\title{
Hybrid iterative method for finding common solutions of generalized mixed equilibrium and fixed point problems
}

\author{
Lu-Chuan Ceng ${ }^{1,2}$, Sy-Ming Guu ${ }^{3 *}$ and Jen-Chih Yao ${ }^{4}$
}

\author{
* Correspondence: \\ iesmguu@saturn.yzu.edu.tw \\ ${ }^{3}$ Department of Business \\ Administration, College of \\ Management, Yuan-Ze University, \\ Chung-Li, Taoyuan Hsien 330, \\ Taiwan \\ Full list of author information is \\ available at the end of the article
}

\begin{abstract}
Recently, Colao et al. (J Math Anal Appl 344:340-352, 2008) introduced a hybrid viscosity approximation method for finding a common element of the set of solutions of an equilibrium problem and the set of fixed points of a finite family of nonexpansive mappings in a real Hilbert space. In this paper, by combining Colao, Marino and Xu's hybrid viscosity approximation method and Yamada's hybrid steepest-descent method, we propose a hybrid iterative method for finding a common element of the set GMEP of solutions of a generalized mixed equilibrium problem and the set $\bigcap_{i=1}^{N}$ Fix $\left(S_{i}\right)$ of fixed points of a finite family of nonexpansive mappings $\left\{S_{i}\right\}_{i=1}^{N}$ in a real Hilbert space. We prove the strong convergence of the proposed iterative algorithm to an element of $\bigcap_{i=1}^{N}$ Fix $\left(S_{i}\right) \cap$ GMEP, which is the unique solution of a variational inequality.

AMS subject classifications: 49J40; 47J20; 47H09.

Keywords: Generalized mixed equilibrium problem, fixed point, nonexpansive mapping, variational inequality, hybrid iterative method.
\end{abstract}

\section{Introduction}

The theory of equilibrium problems has played an important role in the study of a wide class of problems arising in economics, finance, transportation, network and structural analysis, elasticity and optimization, and has numerous applications, including but not limited to problems in economics, game theory, finance, traffic analysis, circuit network analysis and mechanics. The ideas and techniques of this theory are being used in a variety of diverse areas and proved to be productive and innovative. It is remarkable that the variational inequalities and mathematical programming problems can be viewed as a special realization of the abstract equilibrium problems $[1,2]$.

Let $H$ be a real Hilbert space. Throughout this paper, we write $x_{n} \rightarrow x$ to indicate that the sequence $\left\{x_{n}\right\}$ converges weakly to $x$. The $x_{n} \rightarrow x$ indicates that $\left\{x_{n}\right\}$ converges strongly to $x$. Let $C$ be a nonempty closed convex subset of $H$ and $\Theta$ be a bifunction of $C \times C$ into $\mathbf{R}$, where $\mathbf{R}$ is the set of real numbers. The equilibrium problem for $\Theta$ : $C \times C \rightarrow \mathbf{R}$ is to find $\bar{x} \in C$ such that

\section{Springer}

(C) 2012 Ceng et al; licensee Springer. This is an Open Access article distributed under the terms of the Creative Commons Attribution License (http://creativecommons.org/licenses/by/2.0), which permits unrestricted use, distribution, and reproduction in any medium, provided the original work is properly cited. 


$$
\Theta(\bar{x}, y) \geq 0, \quad \forall y \in C .
$$

The set of solutions of problem (1.1) is denoted by $E P(\Theta)$. Given a mapping $T: C \rightarrow H$, let $\Theta(x, y)=\langle T x, y-x\rangle$ for all $x, y \in C$. Then, $z \in E P(\Theta)$ if and only if $\langle T z, y-z\rangle \geq 0$ for all $y \in C$. Numerous problems in physics, optimization, and economics reduce to finding a solution of problem (1.1). Equilibrium problems have been studied extensively [2-18]. Combettes and Hirstoaga [3] introduced an iterative scheme for finding the best approximation to the initial data when $E P(\Theta)$ is nonempty and derived a strong convergence theorem. Very recently, Peng and Yao [4] introduced the following generalized mixed equilibrium problem of finding $\bar{x} \in C$ such that

$$
\Theta(\bar{x}, y)+\varphi(y)-\varphi(\bar{x})+\langle A \bar{x}, y-\bar{x}\rangle \geq 0, \quad \forall y \in C,
$$

where $A: H \rightarrow H$ is a nonlinear mapping, $\phi: C \rightarrow \mathbf{R}$ is a function and $\Theta: C \times C \rightarrow \mathbf{R}$ is a bifunction. The set of solutions of problem (1.2) is denoted by GMEP.

In particular, whenever $A=0$, problem (1.2) reduces to the following mixed equilibrium problem of finding $\bar{x} \in C$ such that

$$
\Theta(\bar{x}, y)+\varphi(y)-\varphi(\bar{x}) \geq 0, \quad \forall y \in C,
$$

which was considered by Ceng and Yao [5]. The set of solutions of this problem is denoted by $M E P$.

Whenever $\phi=0$, problem (1.2) reduces to the following generalized equilibrium problem of finding $\bar{x} \in C$ such that

$$
\Theta(\bar{x}, y)+\langle A \bar{x}, y-\bar{x}\rangle \geq 0, \quad \forall y \in C,
$$

which was introduced and studied by Takahashi and Takahashi [13]. The set of solutions of problem (1.3) is denoted by GEP. Obviously, the generalized equilibrium problem covers the equilibrium problem as a special case. It is assumed in [4] that $\Theta: C \times C \rightarrow \mathbf{R}$ is a bifunction satisfying conditions (H1)-(H4) and $\phi: C \rightarrow \mathbf{R}$ is a lower semicontinuous and convex function with restriction (A1) or (A2), where

(H1) $\Theta(x, x)=0, \forall x \in C$;

(H2) $\Theta$ is monotone, i.e., $\Theta(x, y)+\Theta(y, x) \leq 0, \forall x, y \in C$;

(H3) for each $y \in C, x \mapsto \Theta(x, y)$ is weakly upper semicontinuous;

(H4) for each $x \in C, y \mapsto \Theta(x, y)$ is convex and lower semicontinuous;

(A1) for each $x \in H$ and $r>0$, there exist a bounded subset $D_{x} \subset C$ and $y_{x} \in C$ such that for any $z \in C \backslash D_{x}$,

$$
\Theta\left(z, y_{x}\right)+\varphi\left(y_{x}\right)-\varphi(z)+\frac{1}{r}\left\langle y_{x}-z, z-x\right\rangle<0
$$

(A2) $C$ is a bounded set.

It is worth pointing out that, related iterative methods for solving fixed point problems, variational inequalities and optimization problems can be found in [19-35].

Recall that a $\rho$-Lipschitzian mapping $T: C \rightarrow H$ is a mapping on $C$ such that

$$
\|T x-T y\| \leq \rho\|x-y\|, \quad \forall x, y \in C,
$$

where $\rho \geq 0$ is a constant. In particular, if $\rho \in[0,1)$ then $T$ is called a contraction on $C$; if $\rho=1$ then $T$ is called a nonexpansive mapping on $C$. Denote the set of fixed 
points of $T$ by $\operatorname{Fix}(T)$. It is well known that if $C$ is a nonempty bounded closed convex subset of $H$ and $S: C \rightarrow C$ is nonexpansive, then $\operatorname{Fix}(S) \neq \varnothing$. Let $P_{C}$ be the metric projection of $H$ onto $C$, that is, for every point $x \in H$, there exists a unique nearest point of $C$, denoted by $P_{C} x$, such that $\left\|x-P_{C} x\right\| \leq\|x-y\|$ for all $y \in C$. Recall also that a mapping $A$ of $C$ into $H$ is called

(i) monotone if

$$
\langle A x-A y, x-y\rangle \geq 0, \forall x, y \in C ;
$$

(ii) $\eta$-strongly monotone if there exists a constant $\eta>0$ such that

$$
\langle A x-A y, x-y\rangle \geq \eta\|x-y\|^{2}, \forall x, y \in C ;
$$

(iii) $\delta$-inverse strongly monotone if there exists a constant $\delta>0$ such that

$$
\langle A x-A y, x-y\rangle \geq \delta\|A x-A y\|^{2}, \forall x, y \in C .
$$

Furthermore, let $A$ be a strongly positive bounded linear operator on $H$, that is, there exists a constant $\bar{\gamma}>0$ such that

$$
\langle A x, x\rangle \geq \bar{\gamma}\|x\|^{2}, \quad \forall x \in H .
$$

\subsection{The W-mappings}

The concept of $W$-mappings was introduced in Atsushiba and Takahashi [22]. It is very useful in establishing the convergence of iterative methods for computing a common fixed point of nonlinear mappings (see, for instance, [23,25,27]).

Let $\lambda_{n, 1}, \lambda_{n, 2} \ldots, \lambda_{n, N} \in(0,1], n \geq 1$. Given the nonexpansive mappings $S_{1}, S_{2}, \ldots, S_{N}$ on $H$, Atsushiba and Takahashi defines, for each $n \geq 1$, mappings $U_{n, 1}, U_{n, 2}, \ldots, U_{n, N}$ by

$$
\begin{aligned}
& U_{n, 1}=\lambda_{n, 1} S_{1}+\left(1-\lambda_{n, 1}\right) I \\
& U_{n, 2}=\lambda_{n, 2} S_{2} U_{n, 1}+\left(1-\lambda_{n, 2}\right) I \\
& \vdots \\
& U_{n, N-1}=\lambda_{n, N-1} S_{N-1} U_{n, N-2}+\left(1-\lambda_{n, N-1}\right) I, \\
& W_{n}:=U_{n, N}=\lambda_{n, N} S_{N} U_{n, N-1}+\left(1-\lambda_{n, N}\right) I .
\end{aligned}
$$

The $W_{n}$ is called the $W$-mapping generated by $S_{1}, \ldots, S_{N}$ and $\lambda_{n, 1}, \lambda_{n, 2}, \ldots, \lambda_{n, N}$. Note that Nonexpansivity of $S_{i}$ implies the nonexpansivity of $W_{n}$.

Colao et al. [14] introduced an iterative method for finding a common element of the set of solutions of an equilibrium problem and the set of fixed points of a finite family of nonexpansive mappings in a real Hilbert space $H$. Moreover, they proved the strong convergence of the proposed iterative algorithm.

\subsection{Theorem CMX}

(See [[14], Theorem 3.1]). Let $C$ be a nonempty closed convex subset of a real Hilbert space $H$. Let $\left\{S_{i}\right\}_{i=1}^{N}$ be a finite family of nonexpansive mappings on $H, A$ a strongly positive bounded linear operator on $H$ with coefficient $\bar{\gamma}$ and $f$ an $\alpha$-contraction on $H$ for some $\alpha \in(0,1)$. Moreover, let $\left\{\alpha_{n}\right\}$ be a sequence in $(0,1),\left\{\lambda_{n, i}\right\}_{i=1}^{N}$ a sequence in $[a, b]$ with $0<a \leq b<1,\left\{r_{n}\right\}$ a sequence in $(0, \infty)$ and $\gamma$ and $\beta$ two real numbers such 
that $0<\beta<1$ and $0<\gamma<\bar{\gamma} / \alpha$. Let $\Theta: C \times C \rightarrow \mathbf{R}$ be a bifunction satisfying assumptions (H1)-(H4) and $\cap_{i=1}^{N} \operatorname{Fix}\left(S_{i}\right) \cap E P(\Theta) \neq \varnothing$. For every $n \geq 1$, let $W_{n}$ be the $W$-mapping generated by $S_{1}, \ldots, S_{N}$ and $\lambda_{n, 1}, \lambda_{n, 2}, \ldots, \lambda_{n, N}$. Given $x_{1} \in H$ arbitrarily, suppose the sequences $\left\{x_{n}\right\}$ and $\left\{u_{n}\right\}$ are generated iteratively by

$$
\begin{aligned}
& \Theta\left(u_{n}, y\right)+\frac{1}{r_{n}}\left\langle y-u_{n}, u_{n}-x_{n}\right\rangle \geq 0, \quad \forall y \in C, \\
& x_{n+1}=\alpha_{n} \gamma f\left(x_{n}\right)+\beta x_{n}+\left((1-\beta) I-\alpha_{n} A\right) W_{n} u_{n}, \quad \forall n \geq 1,
\end{aligned}
$$

where the sequences $\left\{\alpha_{n}\right\},\left\{r_{n}\right\}$ and the finite family of sequences $\left\{\lambda_{n, i}\right\}_{i=1}^{N}$ satisfy the conditions:

(i) $\lim _{n \rightarrow \infty} \alpha_{n}=0$ and $\sum_{n=1}^{\infty} \alpha_{n}=\infty$;

(ii) $\lim _{\inf _{n \rightarrow \infty}} r_{n}>0$ and $\lim _{n \rightarrow \infty} r_{n} / r_{n+1}=1\left(\right.$ or $\left.\lim _{n \rightarrow \infty}\left|r_{n+1}-r_{n}\right|=0\right)$;

(iii) $\lim _{n \rightarrow \infty}\left|\lambda_{n, i}-\lambda_{n-1, i}\right|=0$ for every $i \in\{1, \ldots, N\}$.

Then both $\left\{x_{n}\right\}$ and $\left\{u_{n}\right\}$ converge strongly to $x^{*} \in \cap_{i=1}^{N} \operatorname{Fix}\left(S_{i}\right) \cap E P(\Theta)$, which is the unique fixed point of the composite mapping $P_{\cap_{i=1}^{N} \operatorname{Fix}\left(S_{i}\right) \cap E P(\Theta)}(I-A+\gamma f)$, i.e.,

$$
x^{*}=P_{\bigcap_{i=1}^{N} \operatorname{Fix}\left(S_{i}\right) \cap E P(\Theta)}(I-A+\gamma f) x^{*} .
$$

Very recently, Yao et al. [10] relaxed the $\beta$ in Colao, Marino and Xu's iterative scheme (1.6) by a sequence of $\left\{\beta_{n}\right\}$. They showed that if with additional condition 0 $<\lim \inf _{n \rightarrow \infty} \beta_{n} \leq \lim \sup _{n \rightarrow \infty} \beta_{n}<1$ holds, then the sequences $\left\{x_{n}\right\}$ and $\left\{u_{n}\right\}$ generated by (1.6) (but now with $\beta_{n}$ in the place of $\beta$ ) still converge strongly to $x^{*} \in \cap_{i=1}^{N} \operatorname{Fix}\left(S_{i}\right) \cap E P(\Theta)$, which is the unique fixed point of the composite mapping $P_{\cap_{i=1}^{N} \operatorname{Fix}\left(S_{i}\right) \cap E P(\Theta)}(I-A+\gamma f)$, i.e.,

$$
x^{*}=P_{\bigcap_{i=1}^{N} \operatorname{Fix}\left(S_{i}\right) \cap E P(\Theta)}(I-A+\gamma f) x^{*} .
$$

\subsection{Hybrid steepest-descent method}

Let $F: H \rightarrow H$ be a $\kappa$-Lipschitzian and $\eta$-strongly monotone operator with constants $\kappa$, $\eta>0$, and let $T: H \rightarrow H$ be nonexpansive such that $\operatorname{Fix}(T) \neq \varnothing$. Yamada [20] introduced the so-called hybrid steepest-descent method for solving the variational inequality problem: finding $\tilde{x} \in \operatorname{Fix}(T)$ such that

$$
\langle F \tilde{x}, x-\tilde{x}\rangle \geq 0, \forall x \in \operatorname{Fix}(T) .
$$

This method generates a sequence $\left\{x_{n}\right\}$ via the following iterative scheme:

$$
x_{n+1}=T x_{n}-\lambda_{n+1} \mu F\left(T x_{n}\right), \forall n \geq 0,
$$

where $0<\mu<2 \eta / \kappa^{2}$, the initial guess $x_{0} \in H$ is arbitrary and the sequence $\left\{\lambda_{n}\right\}$ in $(0$, 1) satisfies the conditions:

$$
\lambda_{n} \rightarrow 0, \sum_{n=0}^{\infty} \lambda_{n}=\infty \text { and } \sum_{n=0}^{\infty}\left|\lambda_{n+1}-\lambda_{n}\right|<\infty .
$$

A key fact in Yamada's argument is that, for small enough $\lambda>0$, the mapping

$$
T^{\lambda} x:=T x-\lambda \mu F(T x), \forall x \in H
$$


is a contraction, due to the $\kappa$-Lipschitz continuity and $\eta$-strong monotonicity of $F$.

\subsection{Our hybrid model}

In this paper, assume $\Theta: C \times C \rightarrow \mathbf{R}$ is a bifunction satisfying assumptions (H1)-(H4) and $\phi: C \rightarrow \mathbf{R}$ is a lower semicontinuous and convex function with restriction (A1) or (A2). Let the mapping $A$ : $H \rightarrow H$ be $\delta$-inverse strongly monotone, and $\left\{S_{i}\right\}_{i=1}^{N}$ be a finite family of nonexpansive mappings on $H$ such that $\cap_{i=1}^{N} \operatorname{Fix}\left(S_{i}\right) \cap G M E P \neq \emptyset$. Let $F$ : $H \rightarrow H$ be a $\kappa$-Lipschitzian and $\eta$-strongly monotone operator with constants $\kappa, \eta>0$ and $f: H \rightarrow H$ a $\rho$-Lipschitzian mapping with constant $\rho \geq 0$. Let $0<\mu<2 \eta / \kappa^{2}$ and 0 $\leq \gamma \rho<\tau$, where $\tau=1-\sqrt{1-\mu\left(2 \eta-\mu \kappa^{2}\right)}$. By combining Yamada's hybrid steepestdescent method [20] and Colao, Marino and Xu's hybrid viscosity approximation method [14] (see also [10]), we propose the following hybrid iterative method for finding a common element of the set of solutions of generalized mixed equilibrium problem (1.2) and the set of fixed points of finitely many nonexpansive mappings $\left\{S_{i}\right\}_{i=1}^{N}$, that is, for given $x_{1} \in H$ arbitrarily, let $\left\{x_{n}\right\}$ and $\left\{u_{n}\right\}$ be generated iteratively by

$$
\left\{\begin{array}{l}
\Theta\left(u_{n}, y\right)+\varphi(y)-\varphi\left(u_{n}\right)+\left\langle A x_{n}, y-u_{n}\right\rangle+\frac{1}{r_{n}}\left\langle y-u_{n}, u_{n}-x_{n}\right\rangle \geq 0, \quad \forall y \in C, \\
x_{n+1}=\alpha_{n} \gamma f\left(x_{n}\right)+\beta_{n} x_{n}+\left(\left(1-\beta_{n}\right) I-\alpha_{n} \mu F\right) W_{n} u_{n}, \quad \forall n \geq 1,
\end{array}\right.
$$

where $\left\{a_{n}\right\},\left\{\beta_{n}\right\} \subset(0,1),\left\{r_{n}\right\} \subset(0,2 \delta],\left\{\lambda_{n, i}\right\}_{i=1}^{N} \subset[a, b]$ with $0<a \leq b<1$, and $W_{n}$ is the $W$-mapping generated by $S_{1}, \ldots, S_{N}$ and $\lambda_{n, 1}, \lambda_{n, 2}, \ldots, \lambda_{n, N}$. We shall prove that under quite mild hypotheses, both sequences $\left\{x_{n}\right\}$ and $\left\{u_{n}\right\}$ converge strongly to $x^{*} \in \cap_{i=1}^{N} \operatorname{Fix}\left(S_{i}\right) \cap G M E P$, where $x^{*}=P_{\bigcap_{i=1}^{N} \operatorname{Fix}\left(S_{i}\right) \cap G M E P}(I-\mu F+\gamma f) x^{*}$ is a unique solution of the variational inequality:

$$
\left\langle(\mu F-\gamma f) x^{*}, x^{*}-x\right\rangle \leq 0, \forall x \in \bigcap_{i=1}^{N} \operatorname{Fix}\left(S_{i}\right) \cap \text { GMEP. }
$$

Compared with Theorem 3.2 of Yao et al. [10], our Theorem 3.1 improves and extends their Theorem 3.2 [10] in the following aspects:

(i) The contraction $f: H \rightarrow H$ with coefficient $\rho \in(0,1)$ in [[10], Theorem 3.2] is extended to the case of general Lipschitzian mapping $f$ on $H$ with constant $\rho \geq 0$.

(ii) The strongly positive bounded linear operator $A: H \rightarrow H$ with coefficient $\tilde{\gamma}>0$ in [[10], Theorem 3.2] is extended to the case of general $\kappa$-Lipschitzian and $\eta$-strongly monotone operator $F$ : $H \rightarrow H$ with constants $\kappa, \eta>0$.

(iii) The equilibrium problem in [[10], Theorem 3.2] is extended to the case of generalized mixed equilibrium problem (1.2). Obviously, the problem (1.2) is more complicated than their problem (1.1).

(iv) The hybrid viscosity approximation method in [[10], Theorem 3.2] (see also [[14], Theorem 3.1]) is extended to develop our iterative method by virtue of Yamada's hybrid steepest-descent method [20].

\section{Preliminaries}

Let $H$ be a real Hilbert space with inner product $\langle\cdot, \cdot\rangle$, and norm II . II. Let $C$ be a nonempty closed convex subset of $H$. Recall that the metric (or nearest point) projection 
from $H$ onto $C$ is the mapping $P_{C}: H \rightarrow C$ which assigns to each point $x \in H$ the unique point $P_{C} x \in C$ satisfying the property

$$
\left\|x-P_{C} x\right\|=\inf _{y \in C}\|x-y\|=: d(x, C) .
$$

In order to prove our main results in the next section, we need the following lemmas and propositions.

Lemma 2.1 (See [36]). Let $C$ be a nonempty closed convex subset of a real Hilbert space $H$. Given $x \in H$ and $z \in C$, we then have

(i) $z=P_{C} x$ if and only if $\langle x-z, y-z\rangle \leq 0, \forall y \in C$.

(ii) $z=P_{C} x$ if and only if $\|x-z\|^{2} \leq\|x-y\|^{2}-\|y-z\|^{2}, \forall_{y} \in C$.

(iii) $\left\langle P_{C} x-P_{C} y, x-y\right\rangle \geq\left\|P_{C} x-P_{C} y\right\|^{2}, \forall x, y \in H$.

Consequently, $P_{C}$ is nonexpansive and monotone.

Lemma 2.2 (See [5]). Let $C$ be a nonempty closed convex subset of $H$. Let $\Theta: C \times C$ $\rightarrow \mathbf{R}$ be a bifunction satisfying conditions (H1)-(H4) and let $\phi: C \rightarrow \mathbf{R}$ be a lower semicontinuous and convex function. For $r>0$ and $x \in H$, define a mapping $T_{r}^{(\Theta, \varphi)}: H \rightarrow C$ as follows:

$$
T_{r}^{(\Theta, \varphi)}(x)=\left\{z \in C: \Theta(z, y)+\varphi(y)-\varphi(z)+\frac{1}{r}\langle y-z, z-x\rangle \geq 0, \forall y \in C\right\}
$$

for all $x \in H$. Assume that either (A1) or (A2) holds. Then the following assertions hold:

(i) $T_{r}^{(\Theta, \varphi)}(x) \neq \varnothing$ for each $x \in H$ and $T_{r}^{(\Theta, \varphi)}$ is single-valued;

(ii) $T_{r}^{(\Theta, \varphi)}$ is firmly nonexpansive, i.e., for any $x, y \in H$,

$$
\left\|T_{r}^{(\Theta, \varphi)} x-T_{r}^{(\Theta, \varphi)} y\right\|^{2} \leq\left\langle T_{r}^{(\Theta, \varphi)} x-T_{r}^{(\Theta, \varphi)} y, x-y\right\rangle ;
$$

(iii) $\operatorname{Fix}\left(T_{r}^{(\Theta, \varphi)}\right)=\operatorname{MEP}(\Theta, \varphi)$;

(iv) $\operatorname{MEP}(\Theta, \phi)$ is closed and convex.

Remark 2.1. If $\phi=0$, then $T_{r}^{(\Theta, \varphi)}$ is rewritten as $T_{r}^{\Theta}$; if $\Theta=0$ additionally, then $T_{r}^{\Theta}=P_{C}$.

Lemma 2.3 (See [21]). Let $\left\{x_{n}\right\}$ and $\left\{y_{n}\right\}$ be bounded sequences in a Banach space $X$ and let $\left\{\beta_{n}\right\}$ be a sequence in $[0,1]$ with $0<\lim \inf _{n \rightarrow \infty} \beta_{n} \leq \lim \sup _{n \rightarrow \infty} \beta_{n}<1$. Suppose $x_{n+1}=\left(1-\beta_{n}\right) y_{n}+\beta_{n} x_{n}$ for all integers $n \geq 0$ and $\lim \sup _{n \rightarrow \infty}\left(\left\|y_{n+1}-y_{n}\right\|-\| x_{n+1}\right.$ $\left.-x_{n} \|\right) \leq 0$. Then, $\lim _{n \rightarrow \infty}\left\|y_{n}-x_{n}\right\|=0$.

Proposition 2.1 (See [[6], Proposition 2.1]). Let $C, H, \Theta, \phi$ and $T_{r}^{(\Theta, \varphi)}$ be as in Lemma 2.2. Then the following inequality holds:

$$
\left\|T_{s}^{(\Theta, \varphi)} x-T_{t}^{(\Theta, \varphi)} x\right\|^{2} \leq \frac{s-t}{s}\left\langle T_{s}^{(\Theta, \varphi)} x-T_{t}^{(\Theta, \varphi)} x, T_{s}^{(\Theta, \varphi)} x-x\right\rangle
$$

for all $s, t>0$ and $x \in H$.

Lemma 2.4 (See [19]). Let $\left\{a_{n}\right\}$ be a sequence of nonnegative numbers satisfying the condition

$$
a_{n+1} \leq\left(1-\delta_{n}\right) a_{n}+\delta_{n} \sigma_{n}, \forall n \geq 1,
$$


where $\left\{\delta_{n}\right\},\left\{\sigma_{n}\right\}$ are sequences of real numbers such that

(i) $\left\{\delta_{n}\right\} \subset[0,1]$ and $\sum_{n=1}^{\infty} \delta_{n}=\infty$, or equivalently,

$$
\prod_{n=1}^{\infty}\left(1-\delta_{n}\right):=\lim _{n \rightarrow \infty} \prod_{k=1}^{n}\left(1-\delta_{k}\right)=0 ;
$$

(ii) $\lim \sup _{n \rightarrow \infty} \sigma_{n} \leq 0$, or

(ii)' $\sum_{n=1}^{\infty} \delta_{n} \sigma_{n}$ is convergent.

Then $\lim _{n \rightarrow \infty} a_{n}=0$.

We will need the following result concerning the $W$-mapping $W_{n}$ generated by $S_{1}, \ldots$, $S_{N}$ and $\lambda_{n, 1}, \lambda_{n, 2}, \ldots, \lambda_{n, N}$ in (1.5).

Proposition 2.2 (See [23]). Let $C$ be a nonempty closed convex subset of a Banach space $X$. Let $S_{1}, S_{2}, \ldots, S_{N}$ be a finite family of nonexpansive mappings of $C$ into itself such that $\cap_{i=1}^{N} \operatorname{Fix}\left(S_{i}\right) \neq \emptyset$, and let $\lambda_{n, 1}, \lambda_{n, 2}, \ldots, \lambda_{n, N}$ be real numbers such that $0<\lambda_{n, i}$ $\leq b<1$ for $i=1,2, \ldots, N$. For any $n \geq 1$, let $W_{n}$ be the $W$-mapping of $C$ into itself generated by $S_{1}, \ldots, S_{N}$ and $\lambda_{n, 1}, \ldots, \lambda_{n, N}$. If $X$ is strictly convex, then $\operatorname{Fix}\left(W_{n}\right)=\cap_{i=1}^{N} \operatorname{Fix}\left(S_{i}\right)$.

Proposition 2.3 (See [[14], Lemma 2.8]). Let $C$ be a nonempty convex subset of a Banach space. Let $\left\{S_{i}\right\}_{i=1}^{N}$ be a finite family of nonexpansive mappings of $C$ into itself and $\left\{\lambda_{n, i}\right\}_{i=1}^{N}$ be sequences in $[0,1]$ such that $\lambda_{n, i} \rightarrow \lambda_{i}(i=1, \ldots, N)$. Moreover for every integer $n \geq 1$, let $W$ and $W_{n}$ be the $W$-mappings generated by $S_{1}, \ldots, S_{N}$ and $\lambda_{1}, \ldots$, $\lambda_{N}$ and $S_{1}, \ldots, S_{N}$ and $\lambda_{n, 1}, \ldots, \lambda_{n, N}$ respectively. Then for every $x \in C$, it follows that

$$
\lim _{n \rightarrow \infty}\left\|W_{n} x-W x\right\|=0 .
$$

The following two lemmas are the immediate consequences of the inner product on $H$.

Lemma 2.5. For all $x, y \in H$, there holds the inequality

$$
\|x+y\|^{2} \leq\|x\|^{2}+2\langle y, x+y\rangle .
$$

Lemma 2.6 (See [36]). For all $x, y, z \in H$ and $\alpha, \beta, \gamma \in[0,1]$ with $\alpha+\beta+\gamma=1$, there holds the equality

$$
\|\alpha x+\beta y+\gamma z\|^{2}=\alpha\|x\|^{2}+\beta\|\gamma\|^{2}+\gamma\|z\|^{2}-\alpha \beta\|x-\gamma\|^{2}-\beta \gamma\|y-z\|^{2}-\gamma \alpha\|z-x\|^{2} .
$$

The following lemma plays a crucial role in proving strong convergence of our iterative schemes.

Lemma 2.7 (See [[19], Lemma 3.1]). Let $\lambda$ be a number in $(0,1]$ and let $\mu>0$. Let $F$ : $H \rightarrow H$ be an operator on $H$ such that, for some constants $\kappa, \eta>0, F$ is $\kappa$-Lipschitzian and $\eta$-strongly monotone. Associating with a nonexpansive mapping $T: H \rightarrow H$, define the mapping $T^{\lambda}: H \rightarrow H$ by

$$
T^{\lambda} x:=T x-\lambda \mu F(T x), \forall x \in H .
$$

Then $T^{\lambda}$ is a contraction provided $\mu<2 \eta / \kappa^{2}$, that is,

$$
\left\|T^{\lambda} x-T^{\lambda} y\right\| \leq(1-\lambda \tau)\|x-y\|, \forall x, y \in H,
$$

where $\tau=1-\sqrt{1-\mu\left(2 \eta-\mu \kappa^{2}\right)} \in(0,1]$. 
Remark 2.2. Put $F=\frac{1}{2} I$, where $I$ is the identity operator of $H$. Then we have $\mu<2 \eta /$ $\kappa^{2}=4$. Also, put $\mu=2$. Then it is easy to see that $\kappa=\eta=\frac{1}{2}$ and

$$
\tau=1-\sqrt{1-\mu\left(2 \eta-\mu \kappa^{2}\right)}=1-\sqrt{1-2\left(2 \cdot \frac{1}{2}-2\left(\frac{1}{2}\right)^{2}\right)}=1 .
$$

In particular, whenever $\lambda>0$, we have $T^{\lambda} x:=T x-\lambda \mu F(T x)=(1-\lambda) T x$.

\section{Iterative scheme and strong convergence}

In this section, based on Yamada's hybrid steepest-descent method [20] and Colao, Marino and Xu's hybrid viscosity approximation method [14] (see also [10]), we introduce a hybrid iterative method for finding a common element of the set of solutions of generalized mixed equilibrium problem (1.2) and the set of fixed points of finitely many nonexpansive mappings in a real Hilbert space. Moreover, we derive the strong convergence of the proposed iterative algorithm to a common solution of problem (1.2) and the fixed point problem of finitely many nonexpansive mappings.

Theorem 3.1. Let $C$ be a nonempty closed convex subset of a real Hilbert space $H$. Let $\Theta: C \times C \rightarrow \mathbf{R}$ be a bifunction satisfying assumptions (H1)-(H4) and $\phi: C \rightarrow \mathbf{R}$ be a lower semicontinuous and convex function with restriction (A1) or (A2). Let the mapping $A$ : $H \rightarrow H$ be $\delta$-inverse strongly monotone, and $\left\{S_{i}\right\}_{i=1}^{N}$ be a finite family of nonexpansive mappings on $H$ such that $\cap_{i=1}^{N} \operatorname{Fix}\left(S_{i}\right) \cap G M E P \neq \emptyset$. Let $F: H \rightarrow H$ be a $\kappa$-Lipschitzian and $\eta$-strongly monotone operator with constants $\kappa, \eta>0$ and $f: H \rightarrow$ $H$ a $\rho$-Lipschitzian mapping with constant $\rho \geq 0$. Let $0<\mu<2 \eta / \kappa^{2}$ and $0 \leq \gamma \rho<\tau$, where $\tau=1-\sqrt{1-\mu\left(2 \eta-\mu \kappa^{2}\right)}$. Suppose $\left\{a_{n}\right\}$ and $\left\{\beta_{n}\right\}$ are two sequences in $(0,1)$, $\left\{r_{n}\right\}$ is a sequence in $(0,2 \delta]$ and $\left\{\lambda_{n, i}\right\}_{i=1}^{N}$ is a sequence in $[a, b]$ with $0<a \leq b<1$. For every $n \geq 1$, let $W_{n}$ be the $W$-mapping generated by $S_{1}, \ldots, S_{N}$ and $\lambda_{n, 1}, \lambda_{n, 2}, \ldots, \lambda_{n, N}$. Given $x_{1} \in H$ arbitrarily, suppose the sequences $\left\{x_{n}\right\}$ and $\left\{u_{n}\right\}$ are generated iteratively by

$$
\left\{\begin{array}{l}
\Theta\left(u_{n}, y\right)+\varphi(y)-\varphi\left(u_{n}\right)+\left\langle A x_{n}, y-u_{n}\right\rangle+\frac{1}{r_{n}}\left\langle y-u_{n}, u_{n}-x_{n}\right\rangle \geq 0, \quad \forall y \in C, \\
x_{n+1}=\alpha_{n} \gamma f\left(x_{n}\right)+\beta_{n} x_{n}+\left(\left(1-\beta_{n}\right) I-\alpha_{n} \mu F\right) W_{n} u_{n}, \quad \forall n \geq 1,
\end{array}\right.
$$

where the sequences $\left\{a_{n}\right\},\left\{\beta_{n}\right\},\left\{r_{n}\right\}$ and the finite family of sequences $\left\{\lambda_{n, i}\right\}_{i=1}^{N}$ satisfy the conditions:

(i) $\lim _{n \rightarrow \infty} \alpha_{n}=0$ and $\sum_{n=1}^{\infty} \alpha_{n}=\infty$;

(ii) $0<\lim \inf _{n \rightarrow \infty} \beta_{n} \leq \lim \sup _{n \rightarrow \infty} \beta_{n}<1$;

(iii) $0<\lim \inf _{n \rightarrow \infty} r_{n} \leq \lim \sup _{n \rightarrow \infty} r_{n}<2 \delta$ and $\lim _{n \rightarrow \infty}\left(r_{n+1}-r_{n}\right)=0$;

(iv) $\lim _{n \rightarrow \infty}\left(\lambda_{n+1, i}-\lambda_{n, i}\right)=0$ for all $i=1,2, \ldots, N$.

Then both $\left\{x_{n}\right\}$ and $\left\{u_{n}\right\}$ converge strongly to $x^{*} \in \bigcap_{i=1}^{N} \operatorname{Fix}\left(S_{i}\right) \cap$ GMEP , where $x^{*}=P_{\bigcap_{i=1}^{N} \operatorname{Fix}\left(S_{i}\right) \cap G M E P}(I-\mu F+\gamma f) x^{*}$ is a unique solution of the variational inequality:

$$
\left\langle(\mu F-\gamma f) x^{*}, x^{*}-x\right\rangle \leq 0, \forall x \in \bigcap_{i=1}^{N} \operatorname{Fix}\left(S_{i}\right) \cap G M E P .
$$


Proof. Let $Q=P \bigcap_{i=1}^{N} \operatorname{Fix}\left(S_{i}\right) \cap G M E P$. Note that $F: H \rightarrow H$ is a $\kappa$-Lipschitzian and $\eta$ strongly monotone operator with constants $\kappa, \eta>0$ and $f: H \rightarrow H$ is a $\rho$-Lipschitzian mapping with constant $\rho \geq 0$. Then, we have

$$
\begin{aligned}
\|(I-\mu F) x-(I-\mu F) y\|^{2} & =\|x-\gamma\|^{2}-2 \mu\langle x-y, F x-F y\rangle+\mu^{2}\|F x-F y\|^{2} \\
& \leq\left(1-2 \mu \eta+\mu^{2} \kappa^{2}\right)\|x-\gamma\|^{2} \\
& =(1-\tau)^{2}\|x-\gamma\|^{2},
\end{aligned}
$$

where $\tau=1-\sqrt{1-\mu\left(2 \eta-\mu \kappa^{2}\right)}$, and hence

$$
\begin{aligned}
\|Q(I-\mu F+\gamma f)(x)-Q(I-\mu F+\gamma f)(\gamma)\| & \leq\|(I-\mu F+\gamma f)(x)-(I-\mu F+\gamma f)(\gamma)\| \\
& \leq\|(I-\mu F) x-(I-\mu F) y\|+\gamma\|f(x)-f(\gamma)\| \\
& \leq(1-\tau)\|x-\gamma\|+\gamma \rho\|x-\gamma\| \\
& =(1-(\tau-\gamma \rho))\|x-\gamma\|,
\end{aligned}
$$

for all $x, y \in H$. Since $0 \leq \gamma \rho<\tau \leq 1$, it is known that $1-(\tau-\gamma \rho) \in[0,1)$. Therefore, $Q(I-\mu F+\gamma f)$ is a contraction of $H$ into itself, which implies that there exists a unique element $x^{*} \in H$ such that $x^{*}=Q(I-\mu F+\gamma f) x^{*}=P_{\bigcap_{i=1}^{N} \operatorname{Fix}\left(S_{i}\right) \cap G M E P}(I-\mu F+\gamma f) x^{*}$.

From the definition of $T_{r}^{(\Theta, \varphi)}$, we know that $u_{n}=T_{r_{n}}^{(\Theta, \varphi)}\left(x_{n}-r_{n} A x_{n}\right)$. Take $p \in \bigcap_{i=1}^{N} \operatorname{Fix}\left(S_{i}\right) \cap G M E P$ arbitrarily. Since $p=T_{r_{n}}^{(\Theta, \varphi)}\left(p-r_{n} A p\right)=S_{i} p, A$ is $\delta$-inverse strongly monotone and $0<r_{n} \leq 2 \delta$, we deduce that, for any $n \geq 1$,

$$
\begin{aligned}
& \left\|u_{n}-p\right\|^{2}=\left\|T_{r_{n}}^{(\Theta, \varphi)}\left(x_{n}-r_{n} A x_{n}\right)-T_{r_{n}}^{(\Theta, \varphi)}\left(p-r_{n} A p\right)\right\|^{2} \\
& \leq\left\|\left(x_{n}-r_{n} A x_{n}\right)-\left(p-r_{n} A p\right)\right\|^{2} \\
& =\left\|x_{n}-p-r_{n}\left(A x_{n}-A p\right)\right\|^{2} \\
& =\left\|x_{n}-p\right\|^{2}-2 r_{n}\left\langle x_{n}-p, A x_{n}-A p\right\rangle+r_{n}^{2}\left\|A x_{n}-A p\right\|^{2} \\
& \leq\left\|x_{n}-p\right\|^{2}+r_{n}\left(r_{n}-2 \delta\right)\left\|A x_{n}-A p\right\|^{2} \\
& \leq\left\|x_{n}-p\right\|^{2} \text {. }
\end{aligned}
$$

First we will prove that both $\left\{x_{n}\right\}$ and $\left\{u_{n}\right\}$ are bounded.

Indeed, taking into account the control conditions (i) and (ii), we may assume, without loss of generality, that $\alpha_{n} \leq 1-\beta_{n}$ for all $n \geq 1$. Now, by Proposition 2.2 we have $p$ $\in \operatorname{Fix}\left(W_{n}\right)$.

Then utilizing Lemma 2.7, from (3.1) and (3.3) we obtain

$$
\begin{aligned}
& \left\|x_{n+1}-p\right\| \\
& =\left\|\alpha_{n}\left(\gamma f\left(x_{n}\right)-\mu F p\right)+\beta_{n}\left(x_{n}-p\right)+\left(\left(1-\beta_{n}\right) I-\alpha_{n} \mu F\right) W_{n} u_{n}-\left(\left(1-\beta_{n}\right) I-\alpha_{n} \mu F\right) W_{n} p\right\| \\
& \leq \alpha_{n}\left\|\gamma f\left(x_{n}\right)-\mu F p\right\|+\beta_{n}\left\|x_{n}-p\right\|+\left\|\left(\left(1-\beta_{n}\right) I-\alpha_{n} \mu F\right) W_{n} u_{n}-\left(\left(1-\beta_{n}\right) I-\alpha_{n} \mu F\right) W_{n} p\right\| \\
& =\alpha_{n}\left\|\gamma f\left(x_{n}\right)-\mu F p\right\|+\beta_{n}\left\|x_{n}-p\right\|+\left(1-\beta_{n}\right)\left\|\left(I-\frac{\alpha_{n}}{1-\beta_{n}} \mu F\right) W_{n} u_{n}-\left(I-\frac{\alpha_{n}}{1-\beta_{n}} \mu F\right) W_{n} p\right\| \\
& \leq\left(1-\beta_{n}\right)\left(1-\frac{\alpha_{n} \tau}{1-\beta_{n}}\right)\left\|u_{n}-p\right\|+\beta_{n}\left\|x_{n}-p\right\|+\alpha_{n}\left\|\gamma f\left(x_{n}\right)-\mu F p\right\| \\
& \leq\left(1-\beta_{n}-\alpha_{n} \tau\right)\left\|u_{n}-p\right\|+\beta_{n}\left\|x_{n}-p\right\|+\alpha_{n}\left\|\gamma f\left(x_{n}\right)-\mu F p\right\| \\
& \leq\left(1-\alpha_{n} \tau\right)\left\|x_{n}-p\right\|+\alpha_{n} \gamma\left\|f\left(x_{n}\right)-f(p)\right\|+\alpha_{n}\|\gamma f(p)-\mu F p\| \\
& \leq\left(1-\alpha_{n} \tau\right)\left\|x_{n}-p\right\|+\alpha_{n} \gamma \rho\left\|x_{n}-p\right\|+\alpha_{n}\|\gamma f(p)-\mu F p\| \\
& =\left(1-(\tau-\gamma \rho) \alpha_{n}\right)\left\|x_{n}-p\right\|+\alpha_{n}\|\gamma f(p)-\mu F p\| \\
& =\left(1-(\tau-\gamma \rho) \alpha_{n}\right)\left\|x_{n}-p\right\|+(\tau-\gamma \rho) \alpha_{n} \frac{\|\gamma f(p)-\mu F p\|}{\tau-\gamma \rho} \\
& \leq \max \left\{\left\|x_{n}-p\right\|, \frac{\|\gamma f(p)-\mu F p\|}{\tau-\gamma \rho}\right\} .
\end{aligned}
$$


It follows from (3.4) and induction that

$$
\left\|x_{n}-p\right\| \leq \max \left\{\left\|x_{0}-p\right\|, \frac{\|\gamma f(p)-\mu F p\|}{\tau-\gamma \rho}\right\}, \forall n \geq 1
$$

Therefore $\left\{x_{n}\right\}$ is bounded. We also obtain that $\left\{u_{n}\right\},\left\{A x_{n}\right\},\left\{W_{n} u_{n}\right\}$ and $\left\{f\left(x_{n}\right)\right\}$ are all bounded. We shall use $M$ to denote the possible different constants appearing in the following reasoning.

Next, we show that $\left\|x_{n+1}-x_{n}\right\| \rightarrow 0$.

Indeed, set $x_{n+1}=\beta_{n} x_{n}+\left(1-\beta_{n}\right) z_{n}$ for all $n \geq 1$. Then from the definition of $z_{n}$ we obtain

$$
\begin{aligned}
z_{n+1}-z_{n}= & \frac{x_{n+2}-\beta_{n+1} x_{n+1}}{1-\beta_{n+1}}-\frac{x_{n+1}-\beta_{n} x_{n}}{1-\beta_{n}} \\
= & \frac{\alpha_{n+1} \gamma f\left(x_{n+1}\right)+\left(\left(1-\beta_{n+1}\right) I-\alpha_{n+1} \mu F\right) W_{n+1} u_{n+1}}{1-\beta_{n+1}}-\frac{\alpha_{n} \gamma f\left(x_{n}\right)+\left(\left(1-\beta_{n}\right) I-\alpha_{n} \mu F\right) W_{n} u_{n}}{1-\beta_{n}} \\
= & \frac{\alpha_{n+1}}{1-\beta_{n+1}} \gamma f\left(x_{n+1}\right)-\frac{\alpha_{n}}{1-\beta_{n}} \gamma f\left(x_{n}\right)+W_{n+1} u_{n+1} \\
& -W_{n} u_{n}+\frac{\alpha_{n}}{1-\beta_{n}} \mu F W_{n} u_{n}-\frac{\alpha_{n+1}}{1-\beta_{n+1}} \mu F W_{n+1} u_{n+1} \\
= & \frac{\alpha_{n+1}}{1-\beta_{n+1}}\left[\gamma f\left(x_{n+1}\right)-\mu F W_{n+1} u_{n+1}\right]+\frac{\alpha_{n}}{1-\beta_{n}}\left[\mu F W_{n} u_{n}-\gamma f\left(x_{n}\right)\right] \\
& +W_{n+1} u_{n+1}-W_{n+1} u_{n}+W_{n+1} u_{n}-W_{n} u_{n} .
\end{aligned}
$$

It follows that

$$
\begin{aligned}
\left\|z_{n+1}-z_{n}\right\|-\left\|x_{n+1}-x_{n}\right\| \leq & \frac{\alpha_{n+1}}{1-\beta_{n+1}}\left(\gamma\left\|f\left(x_{n+1}\right)\right\|+\mu\left\|F W_{n+1} u_{n+1}\right\|\right) \\
& +\frac{\alpha_{n}}{1-\beta_{n}}\left(\mu\left\|F W_{n} u_{n}\right\|+\gamma\left\|f\left(x_{n}\right)\right\|\right)+\| W_{n+1} u_{n+1} \\
& -W_{n+1} u_{n}\|+\| W_{n+1} u_{n}-W_{n} u_{n}\|-\| x_{n+1}-x_{n} \| \\
\leq & \frac{\alpha_{n+1}}{1-\beta_{n+1}}\left(\gamma\left\|f\left(x_{n+1}\right)\right\|+\mu\left\|F W_{n+1} u_{n+1}\right\|\right) \\
& +\frac{\alpha_{n}}{1-\beta_{n}}\left(\mu\left\|F W_{n} u_{n}\right\|+\gamma\left\|f\left(x_{n}\right)\right\|\right)+\left\|W_{n+1} u_{n}-W_{n} u_{n}\right\| \\
& +\left\|u_{n+1}-u_{n}\right\|-\left\|x_{n+1}-x_{n}\right\| .
\end{aligned}
$$

From (1.5), since $S_{i}$ and $U_{n, i}$ for all $i=1,2, \ldots, N$ are nonexpansive,

$$
\begin{aligned}
\| & W_{n+1} u_{n}-W_{n} u_{n} \| \\
= & \left\|\lambda_{n+1, N} S_{N} U_{n+1, N-1} u_{n}+\left(1-\lambda_{n+1, N}\right) u_{n}-\lambda_{n, N} S_{N} U_{n, N-1} u_{n}-\left(1-\lambda_{n, N}\right) u_{n}\right\| \\
\leq & \left|\lambda_{n+1, N}-\lambda_{n, N}\right|\left\|u_{n}\right\|+\left\|\lambda_{n+1, N} S_{N} U_{n+1, N-1} u_{n}-\lambda_{n, N} S_{N} U_{n, N-1} u_{n}\right\| \\
\leq & \left|\lambda_{n+1, N}-\lambda_{n, N}\right|\left\|u_{n}\right\|+\left\|\lambda_{n+1, N}\left(S_{N} U_{n+1, N-1} u_{n}-S_{N} U_{n, N-1} u_{n}\right)\right\| \\
& +\left|\lambda_{n+1, N}-\lambda_{n, N}\right|\left\|S_{N} U_{n, N-1} u_{n}\right\| \\
\leq & 2 M\left|\lambda_{n+1, N}-\lambda_{n, N}\right|+\lambda_{n+1, N}\left\|U_{n+1, N-1} u_{n}-U_{n, N-1} u_{n}\right\| .
\end{aligned}
$$

Again, from (1.5),

$$
\begin{aligned}
\| & U_{n+1, N-1} u_{n}-U_{n, N-1} u_{n} \| \\
= & \left\|\lambda_{n+1, N-1} S_{N-1} U_{n+1, N-2} u_{n}+\left(1-\lambda_{n+1, N-1}\right) u_{n}-\lambda_{n, N-1} S_{N-1} U_{n, N-2} u_{n}-\left(1-\lambda_{n, N-1}\right) u_{n}\right\| \\
\leq & \left|\lambda_{n+1, N-1}-\lambda_{n, N-1}\right|\left\|u_{n}\right\|+\left\|\lambda_{n+1, N-1} S_{N-1} U_{n+1, N-2} u_{n}-\lambda_{n, N-1} S_{N-1} U_{n, N-2} u_{n}\right\| \\
\leq & \left|\lambda_{n+1, N-1}-\lambda_{n, N-1}\right|\left\|u_{n}\right\|+\lambda_{n+1, N-1}\left\|S_{N-1} U_{n+1, N-2} u_{n}-S_{N-1} U_{n, N-2} u_{n}\right\| \\
& +\left|\lambda_{n+1, N-1}-\lambda_{n, N-1}\right| M \\
\leq & 2 M\left|\lambda_{n+1, N-1}-\lambda_{n, N-1}\right|+\lambda_{n+1, N-1}\left\|U_{n+1, N-2} u_{n}-U_{n, N-2} u_{n}\right\| \\
\leq & 2 M\left|\lambda_{n+1, N-1}-\lambda_{n, N-1}\right|+\left\|U_{n+1, N-2} u_{n}-U_{n, N-2} u_{n}\right\| .
\end{aligned}
$$


Therefore, we have

$$
\begin{aligned}
& \left\|U_{n+1, N-1} u_{n}-U_{n, N-1} u_{n}\right\| \\
& \leq 2 M\left|\lambda_{n+1, N-1}-\lambda_{n, N-1}\right|+2 M\left|\lambda_{n+1, N-2}-\lambda_{n, N-2}\right|+\left\|U_{n+1, N-3} u_{n}-U_{n, N-3} u_{n}\right\| \\
& \leq 2 M \sum_{i=2}^{N-1}\left|\lambda_{n+1, i}-\lambda_{n, i}\right|+\left\|U_{n+1,1} u_{n}-U_{n, 1} u_{n}\right\| \\
& =\left\|\lambda_{n+1,1} S_{1} u_{n}+\left(1-\lambda_{n+1,1}\right) u_{n}-\lambda_{n, 1} S_{1} u_{n}-\left(1-\lambda_{n, 1}\right) u_{n}\right\|+2 M \sum_{i=2}^{N-1}\left|\lambda_{n+1, i}-\lambda_{n, i}\right|,
\end{aligned}
$$

and then

$$
\begin{aligned}
& \left\|U_{n+1, N-1} u_{n}-U_{n, N-1} u_{n}\right\| \\
& \leq\left|\lambda_{n+1,1}-\lambda_{n, 1}\right|\left\|u_{n}\right\|+\left\|\lambda_{n+1,1} S_{1} u_{n}-\lambda_{n, 1} S_{1} u_{n}\right\|+2 M \sum_{i=2}^{N-1}\left|\lambda_{n+1, i}-\lambda_{n, i}\right| \\
& \leq 2 M \sum_{i=1}^{N-1}\left|\lambda_{n+1, i}-\lambda_{n, i}\right|
\end{aligned}
$$

Substituting (3.8) into (3.6), we have

$$
\begin{aligned}
\left\|W_{n+1} u_{n}-W_{n} u_{n}\right\| & \leq 2 M\left|\lambda_{n+1, N}-\lambda_{n, N}\right|+2 \lambda_{n+1, N} M \sum_{i=1}^{N-1}\left|\lambda_{n+1, i}-\lambda_{n, i}\right| \\
& \leq 2 M \sum_{i=1}^{N}\left|\lambda_{n+1, i}-\lambda_{n, i}\right| .
\end{aligned}
$$

On the other hand, utilizing the $\delta$-inverse strongly monotonicity of $A$ we have

$$
\begin{aligned}
\left\|\left(x_{n+1}-r_{n+1} A x_{n+1}\right)-\left(x_{n}-r_{n} A x_{n}\right)\right\| & =\left\|x_{n+1}-x_{n}-r_{n+1}\left(A x_{n+1}-A x_{n}\right)+\left(r_{n}-r_{n+1}\right) A x_{n}\right\| \\
& \leq\left\|x_{n+1}-x_{n}-r_{n+1}\left(A x_{n+1}-A x_{n}\right)\right\|+\left|r_{n+1}-r_{n}\right|\left\|A x_{n}\right\| \\
& \leq\left\|x_{n+1}-x_{n}\right\|+\left|r_{n+1}-r_{n}\right|\left\|A x_{n}\right\|,
\end{aligned}
$$

Since $u_{n}=T_{r_{n}}^{(\Theta, \varphi)}\left(x_{n}-r_{n} A x_{n}\right)$ and $u_{n+1}=T_{r_{n+1}}^{(\Theta, \varphi)}\left(x_{n+1}-r_{n+1} A x_{n+1}\right)$, we get

$$
\begin{aligned}
& \left\|u_{n+1}-u_{n}\right\| \\
& =\left\|T_{r_{n+1}}^{(\Theta, \varphi)}\left(x_{n+1}-r_{n+1} A x_{n+1}\right)-T_{r_{n}}^{(\Theta, \varphi)}\left(x_{n}-r_{n} A x_{n}\right)\right\| \\
& =\left\|T_{r_{n+1}}^{(\Theta, \varphi)}\left(x_{n+1}-r_{n+1} A x_{n+1}\right)-T_{r_{n+1}}^{(\Theta, \varphi)}\left(x_{n}-r_{n} A x_{n}\right)+T_{r_{n+1}}^{(\Theta, \varphi)}\left(x_{n}-r_{n} A x_{n}\right)-T_{r_{n}}^{(\Theta, \varphi)}\left(x_{n}-r_{n} A x_{n}\right)\right\| \\
& \leq\left\|T_{r_{n+1}}^{(\Theta, \varphi)}\left(x_{n+1}-r_{n+1} A x_{n+1}\right)-T_{r_{n+1}}^{(\Theta, \varphi)}\left(x_{n}-r_{n} A x_{n}\right)\right\|+\left\|T_{r_{n+1}}^{(\Theta, \varphi)}\left(x_{n}-r_{n} A x_{n}\right)-T_{r_{n}}^{(\Theta, \varphi)}\left(x_{n}-r_{n} A x_{n}\right)\right\| \\
& \leq\left\|\left(x_{n+1}-r_{n+1} A x_{n+1}\right)-\left(x_{n}-r_{n} A x_{n}\right)\right\|+\left\|T_{r_{n+1}}^{(\Theta, \varphi)}\left(x_{n}-r_{n} A x_{n}\right)-T_{r_{n}}^{(\Theta, \varphi)}\left(x_{n}-r_{n} A x_{n}\right)\right\| \\
& \leq\left\|x_{n+1}-x_{n}\right\|+\left|r_{n+1}-r_{n}\right|\left\|A x_{n}\right\|+\left\|T_{r_{n+1}}^{(\Theta, \varphi)}\left(x_{n}-r_{n} A x_{n}\right)-T_{r_{n}}^{(\Theta, \varphi)}\left(x_{n}-r_{n} A x_{n}\right)\right\|,
\end{aligned}
$$

Using (3.9) and (3.11) in (3.5), we get

$$
\begin{aligned}
& \left\|z_{n+1}-z_{n}\right\|-\left\|x_{n+1}-x_{n}\right\| \\
& \leq \frac{\alpha_{n+1}}{1-\beta_{n+1}}\left(\gamma\left\|f\left(x_{n+1}\right)\right\|+\mu\left\|F W_{n+1} u_{n+1}\right\|\right)+\frac{\alpha_{n}}{1-\beta_{n}}\left(\mu\left\|F W_{n} u_{n}\right\|+\gamma\left\|f\left(x_{n}\right)\right\|\right) \\
& \quad+2 M \sum_{i=1}^{N}\left|\lambda_{n+1, i}-\lambda_{n, i}\right|+\left\|x_{n+1}-x_{n}\right\|+\left|r_{n+1}-r_{n}\right|\left\|A x_{n}\right\| \\
& \quad+\left\|T_{r_{n+1}}^{(\Theta)}\left(x_{n}-r_{n} A x_{n}\right)-T_{r_{n}}^{(\Theta, \varphi)}\left(x_{n}-r_{n} A x_{n}\right)\right\|-\left\|x_{n+1}-x_{n}\right\| \\
& =\frac{\alpha_{n+1}}{1-\beta_{n+1}}\left(\gamma\left\|f\left(x_{n+1}\right)\right\|+\mu\left\|F W_{n+1} u_{n+1}\right\|\right)+\frac{\alpha_{n}}{1-\beta_{n}}\left(\mu\left\|F W_{n} u_{n}\right\|+\gamma\left\|f\left(x_{n}\right)\right\|\right) \\
& \quad+2 M \sum_{i=1}^{N}\left|\lambda_{n+1, i}-\lambda_{n, i}\right|+\left|r_{n+1}-r_{n}\right|\left\|A x_{n}\right\| \\
& \quad+\left\|T_{r_{n+1}}^{(\Theta, \varphi)}\left(x_{n}-r_{n} A x_{n}\right)-T_{r_{n}}^{(\Theta, \varphi)}\left(x_{n}-r_{n} A x_{n}\right)\right\| .
\end{aligned}
$$


Note that $0<\lim \inf _{n \rightarrow \infty} r_{n} \leq \lim \sup _{n \rightarrow \infty} r_{n}<2 \delta$ and $\lim _{n \rightarrow \infty}\left(r_{n+1}-r_{n}\right)=0$. Then utilizing Proposition 2.1 we have

$$
\lim _{n \rightarrow \infty}\left\|T_{r_{n+1}}^{(\Theta, \varphi)}\left(x_{n}-r_{n} A x_{n}\right)-T_{r_{n}}^{(\Theta, \varphi)}\left(x_{n}-r_{n} A x_{n}\right)\right\|=0 .
$$

Consequently, it follows from (3.13) and conditions (i), (iii), (iv) that

$$
\begin{aligned}
& \underset{n \rightarrow \infty}{\limsup }\left(\left\|z_{n+1}-z_{n}\right\|-\left\|x_{n+1}-x_{n}\right\|\right) \\
& \leq \limsup _{n \rightarrow \infty}\left\{\frac{\alpha_{n+1}}{1-\beta_{n+1}}\left(\gamma\left\|f\left(x_{n+1}\right)\right\|+\mu\left\|F W_{n+1} u_{n+1}\right\|\right)+\frac{\alpha_{n}}{1-\beta_{n}}\left(\mu\left\|F W_{n} u_{n}\right\|+\gamma\left\|f\left(x_{n}\right)\right\|\right)\right. \\
& \left.\quad+2 M \sum_{i=1}^{N}\left|\lambda_{n+1, i}-\lambda_{n, i}\right|+\left|r_{n+1}-r_{n}\right|\left\|A x_{n}\right\|+\left\|T_{r_{n+1}}^{(\Theta, \varphi)}\left(x_{n}-r_{n} A x_{n}\right)-T_{r_{n}}^{(\Theta, \varphi)}\left(x_{n}-r_{n} A x_{n}\right)\right\|\right\} \\
& =0 .
\end{aligned}
$$

Hence by Lemma 2.3 we have

$$
\lim _{n \rightarrow \infty}\left\|z_{n}-x_{n}\right\|=0 \text {. }
$$

\section{Consequently}

$$
\lim _{n \rightarrow \infty}\left\|x_{n+1}-x_{n}\right\|=\lim _{n \rightarrow \infty}\left(1-\beta_{n}\right)\left\|z_{n}-x_{n}\right\|=0 .
$$

From (3.11), (3.13), (3.14) and condition (iii) we have

$$
\lim _{n \rightarrow \infty}\left\|u_{n+1}-u_{n}\right\|=0 .
$$

Since $x_{n+1}=a_{n} \gamma f\left(x_{n}\right)+\beta_{n} x_{n}+\left(\left(1-\beta_{n}\right) I-a_{n} \mu F\right) W_{n} u_{n}$, we have

$$
\begin{aligned}
\left\|x_{n}-W_{n} u_{n}\right\| & \leq\left\|x_{n}-x_{n+1}\right\|+\left\|x_{n+1}-W_{n} u_{n}\right\| \\
& \leq\left\|x_{n}-x_{n+1}\right\|+\alpha_{n}\left\|\gamma f\left(x_{n}\right)-\mu F W_{n} u_{n}\right\|+\beta_{n}\left\|x_{n}-W_{n} u_{n}\right\|,
\end{aligned}
$$

that is

$$
\left\|x_{n}-W_{n} u_{n}\right\| \leq \frac{1}{1-\beta_{n}}\left\|x_{n}-x_{n+1}\right\|+\frac{\alpha_{n}}{1-\beta_{n}}\left\|\gamma f\left(x_{n}\right)-\mu F W_{n} u_{n}\right\| .
$$

It follows that

$$
\lim _{n \rightarrow \infty}\left\|x_{n}-W_{n} u_{n}\right\|=0 .
$$

On the other hand, from (3.3) and (3.4) we get

$$
\begin{aligned}
\left\|x_{n+1}-p\right\|^{2} \leq & {\left[\left(1-\beta_{n}-\alpha_{n} \tau\right)\left\|u_{n}-p\right\|+\beta_{n}\left\|x_{n}-p\right\|+\alpha_{n}\left\|\gamma f\left(x_{n}\right)-\mu F p\right\|\right]^{2} } \\
\leq & \left(1-\beta_{n}-\alpha_{n} \tau\right)\left\|u_{n}-p\right\|^{2}+\beta_{n}\left\|x_{n}-p\right\|^{2}+\frac{\alpha_{n}}{\tau}\left\|\gamma f\left(x_{n}\right)-\mu F p\right\|^{2} \\
\leq & \left(1-\beta_{n}-\alpha_{n} \tau\right)\left[\left\|x_{n}-p\right\|^{2}+r_{n}\left(r_{n}-2 \delta\right)\left\|A x_{n}-A p\right\|^{2}\right]+\beta_{n}\left\|x_{n}-p\right\|^{2} \\
& +\frac{\alpha_{n}}{\tau}\left\|\gamma f\left(x_{n}\right)-\mu F p\right\|^{2} \\
= & \left(1-\alpha_{n} \tau\right)\left\|x_{n}-p\right\|^{2}+r_{n}\left(r_{n}-2 \delta\right)\left(1-\beta_{n}-\alpha_{n} \tau\right)\left\|A x_{n}-A p\right\|^{2} \\
& +\frac{\alpha_{n}}{\tau}\left\|\gamma f\left(x_{n}\right)-\mu F p\right\|^{2} \\
\leq & \left\|x_{n}-p\right\|^{2}+r_{n}\left(r_{n}-2 \delta\right)\left(1-\beta_{n}-\alpha_{n} \tau\right)\left\|A x_{n}-A p\right\|^{2}+\frac{\alpha_{n}}{\tau}\left\|\gamma f\left(x_{n}\right)-\mu F p\right\|^{2},
\end{aligned}
$$

and hence

$$
\begin{aligned}
& r_{n}\left(2 \delta-r_{n}\right)\left(1-\beta_{n}-\alpha_{n} \tau\right)\left\|A x_{n}-A p\right\|^{2} \\
& \leq\left\|x_{n}-p\right\|^{2}-\left\|x_{n+1}-p\right\|^{2}+\frac{\alpha_{n}}{\tau}\left\|\gamma f\left(x_{n}\right)-\mu F p\right\|^{2} \\
& =\left(\left\|x_{n}-p\right\|+\left\|x_{n+1}-p\right\|\right)\left\|x_{n}-x_{n+1}\right\|+\frac{\alpha_{n}}{\tau}\left\|\gamma f\left(x_{n}\right)-\mu F p\right\|^{2} .
\end{aligned}
$$


Obviously, conditions (i), (ii), (iii) guarantee that $\alpha_{n} \rightarrow 0,0<\lim _{n \rightarrow \infty} \beta_{n} \leq \lim$ $\sup _{n \rightarrow \infty} \beta_{n}<1$ and $0<\lim _{\inf _{n \rightarrow \infty}} r_{n} \leq \lim \sup _{n \rightarrow \infty} r_{n}<2 \delta$. Thus from I| $x_{n}-x_{n+1} \|$ $\rightarrow 0$ we conclude that

$$
\lim _{n \rightarrow \infty}\left\|A x_{n}-A p\right\|=0 .
$$

Note that $T_{r}^{(\Theta, \varphi)}$ is firmly nonexpansive. Hence we have

$$
\begin{aligned}
& \left\|u_{n}-p\right\|^{2} \\
& =\left\|T_{r_{n}}^{(\Theta, \varphi)}\left(x_{n}-r_{n} A x_{n}\right)-T_{r_{n}}^{(\Theta, \varphi)}\left(p-r_{n} A p\right)\right\|^{2} \\
& \leq\left\langle\left(x_{n}-r_{n} A x_{n}\right)-\left(p-r_{n} A p\right), u_{n}-p\right\rangle \\
& =\frac{1}{2}\left[\left\|\left(x_{n}-r_{n} A x_{n}\right)-\left(p-r_{n} A p\right)\right\|^{2}+\left\|u_{n}-p\right\|^{2}-\left\|\left(x_{n}-r_{n} A x_{n}\right)-\left(p-r_{n} A p\right)-\left(u_{n}-p\right)\right\|^{2}\right] \\
& \leq \frac{1}{2}\left[\left\|x_{n}-p\right\|^{2}+\left\|u_{n}-p\right\|^{2}-\left\|x_{n}-u_{n}-r_{n}\left(A x_{n}-A p\right)\right\|^{2}\right] \\
& =\frac{1}{2}\left[\left\|x_{n}-p\right\|^{2}+\left\|u_{n}-p\right\|^{2}-\left\|x_{n}-u_{n}\right\|^{2}+2 r_{n}\left\langle A x_{n}-A p, x_{n}-u_{n}\right\rangle-r_{n}^{2}\left\|A x_{n}-A p\right\|^{2}\right],
\end{aligned}
$$

which implies that

$$
\left\|u_{n}-p\right\|^{2} \leq\left\|x_{n}-p\right\|^{2}-\left\|x_{n}-u_{n}\right\|^{2}+2 r_{n}\left\|A x_{n}-A p\right\|\left\|x_{n}-u_{n}\right\| .
$$

Therefore, utilizing Lammas 2.5 and 2.7 we deduce from (3.17) that

$$
\begin{aligned}
& \left\|x_{n+1}-p\right\|^{2} \\
& =\left\|\alpha_{n}\left(\gamma f\left(x_{n}\right)-\mu F p\right)+\beta_{n}\left(x_{n}-W_{n} u_{n}\right)+\left(I-\alpha_{n} \mu F\right) W_{n} u_{n}-\left(I-\alpha_{n} \mu F\right) W_{n} p\right\|^{2} \\
& \leq\left\|\left(I-\alpha_{n} \mu F\right) W_{n} u_{n}-\left(I-\alpha_{n} \mu F\right) W_{n} p+\beta_{n}\left(x_{n}-W_{n} u_{n}\right)\right\|^{2}+2 \alpha_{n}\left\langle\gamma f\left(x_{n}\right)-\mu F p, x_{n+1}-p\right\rangle \\
& \leq\left[\left\|\left(I-\alpha_{n} \mu F\right) W_{n} u_{n}-\left(I-\alpha_{n} \mu F\right) W_{n} p+\beta_{n}\right\| x_{n}-W_{n} u_{n} \|\right]^{2}+2 \alpha_{n}\left\|\gamma f\left(x_{n}\right)-\mu F p\right\|\left\|x_{n+1}-p\right\| \\
& \leq\left[\left(1-\alpha_{n} \tau\right)\left\|u_{n}-p\right\|+\beta_{n}\left\|x_{n}-W_{n} u_{n}\right\|\right]^{2}+2 \alpha_{n}\left\|\gamma f\left(x_{n}\right)-\mu F p\right\|\left\|x_{n+1}-p\right\| \\
& \leq\left(\left\|u_{n}-p\right\|+\left\|x_{n}-W_{n} u_{n}\right\|\right)^{2}+2 \alpha_{n}\left\|\gamma f\left(x_{n}\right)-\mu F p\right\|\left\|x_{n+1}-p\right\| \\
& =\left\|u_{n}-p\right\|^{2}+\left\|x_{n}-W_{n} u_{n}\right\|^{2}+2\left\|u_{n}-p\right\|\left\|x_{n}-W_{n} u_{n}\right\|+2 \alpha_{n}\left\|\gamma f\left(x_{n}\right)-\mu F p\right\|\left\|x_{n+1}-p\right\| \\
& \leq\left\|x_{n}-p\right\|^{2}-\left\|x_{n}-u_{n}\right\|^{2}+2 r_{n}\left\|A x_{n}-A p\right\|\left\|x_{n}-u_{n}\right\|+\left\|x_{n}-W_{n} u_{n}\right\|^{2} \\
& \quad+2\left\|u_{n}-p\right\|\left\|x_{n}-W_{n} u_{n}\right\|+2 \alpha_{n}\left\|\gamma f\left(x_{n}\right)-\mu F p\right\|\left\|x_{n+1}-p\right\| .
\end{aligned}
$$

Then we have

$$
\begin{aligned}
\left\|x_{n}-u_{n}\right\|^{2} \leq & \left\|x_{n}-p\right\|^{2}-\left\|x_{n+1}-p\right\|^{2}+2 r_{n}\left\|A x_{n}-A p\right\|\left\|x_{n}-u_{n}\right\|+\left\|x_{n}-W_{n} u_{n}\right\|^{2} \\
& +2\left\|u_{n}-p\right\|\left\|x_{n}-W_{n} u_{n}\right\|+2 \alpha_{n}\left\|\gamma f\left(x_{n}\right)-\mu F p\right\|\left\|x_{n+1}-p\right\| \\
\leq & \left(\left\|x_{n}-p\right\|+\left\|x_{n+1}-p\right\|\right)\left\|x_{n}-x_{n+1}\right\|+2 r_{n}\left\|A x_{n}-A p\right\|\left\|x_{n}-u_{n}\right\| \\
& +\left\|x_{n}-W_{n} u_{n}\right\|^{2}+2\left\|u_{n}-p\right\|\left\|x_{n}-W_{n} u_{n}\right\|+2 \alpha_{n}\left\|\gamma f\left(x_{n}\right)-\mu F p\right\|\left\|x_{n+1}-p\right\| .
\end{aligned}
$$

So, from (3.14)-(3.16) and $\alpha_{n} \rightarrow 0$, we have

$$
\lim _{n \rightarrow \infty}\left\|x_{n}-u_{n}\right\|=0 .
$$

Since

$$
\left\|W_{n} u_{n}-u_{n}\right\| \leq\left\|W_{n} u_{n}-x_{n}\right\|+\left\|x_{n}-u_{n}\right\|,
$$

we also have

$$
\lim _{n \rightarrow \infty}\left\|W_{n} u_{n}-u_{n}\right\|=0 .
$$

Next, let us show that

$$
\limsup _{n \rightarrow \infty}\left\langle(\mu F-\gamma f) x^{*}, x^{*}-x_{n}\right\rangle \leq 0,
$$


where $x^{*}=P_{\bigcap_{i=1}^{N} \operatorname{Fix}\left(S_{i}\right) \cap G M E P}(I-\mu F+\gamma f) x^{*}$ is a unique solution of the variational inequality (3.2). To show this, we can choose a subsequence $\left\{u_{n_{i}}\right\}$ of $\left\{u_{n}\right\}$ such that

$$
\limsup _{n \rightarrow \infty}\left\langle(\mu F-\gamma f) x^{*}, x^{*}-u_{n}\right\rangle=\lim _{i \rightarrow \infty}\left\langle(\mu F-\gamma f) x^{*}, x^{*}-u_{n_{i}}\right\rangle .
$$

Since $\left\{u_{n_{i}}\right\}$ is bounded, there exists a subsequence $\left\{u_{i j}\right\}$ of $\left\{u_{n_{i}}\right\}$ which converges weakly to $w$. Without loss of generality, we may assume that $u_{n_{i}} \rightarrow w$. From $\| W_{n} u_{n}$ $u_{n} \| \rightarrow 0$, we obtain $W_{n} u_{n_{i}} \rightarrow w$. Now we show that $w \in G M E P$. From $u_{n}=T_{r_{n}}^{(\Theta, \varphi)}\left(x_{n}-r_{n} A x_{n}\right)$, we know that

$$
\Theta\left(u_{n}, y\right)+\varphi(y)-\varphi\left(u_{n}\right)+\left\langle A x_{n}, y-u_{n}\right\rangle+\frac{1}{r_{n}}\left\langle y-u_{n}, u_{n}-x_{n}\right\rangle \geq 0, \forall y \in C .
$$

From $(\mathrm{H} 2)$ it follows that

$$
\varphi(y)-\varphi\left(u_{n}\right)+\left\langle A x_{n}, y-u_{n}\right\rangle+\frac{1}{r_{n}}\left\langle y-u_{n}, u_{n}-x_{n}\right\rangle \geq \Theta\left(y, u_{n}\right), \forall y \in C .
$$

Replacing $n$ by $n_{i}$, we have

$$
\varphi(y)-\varphi\left(u_{n_{i}}\right)+\left\langle A x_{n_{i}}, y-u_{n_{i}}\right\rangle+\left\langle y-u_{n_{i}}, \frac{u_{n_{i}}-x_{n_{i}}}{r_{n_{i}}}\right\rangle \geq \Theta\left(y, u_{n_{i}}\right), \forall y \in C .
$$

Put $u_{t}=t y+(1-t) w$ for all $t \in(0,1]$ and $y \in C$. Then, we have $u_{t} \in C$. So, from (3.18) we have

$$
\begin{aligned}
\left\langle u_{t}-u_{n_{i}}, A u_{t}\right\rangle \geq & \left\langle u_{t}-u_{n_{i}}, A u_{t}\right\rangle-\varphi\left(u_{t}\right)+\varphi\left(u_{n_{i}}\right)-\left\langle u_{t}-u_{n_{i}}, A x_{n_{i}}\right\rangle \\
& -\left\langle u_{t}-u_{n_{i}}, \frac{u_{n_{n}}-x_{n_{i}}}{r_{n_{i}}}\right\rangle+\Theta\left(u_{t}, u_{n_{i}}\right) \\
= & \left\langle u_{t}-u_{n_{i}}, A u_{t}-A u_{n_{i}}\right\rangle+\left\langle u_{t}-u_{n_{i}}, A u_{n_{i}}-A x_{n_{i}}\right\rangle-\varphi\left(u_{t}\right)+\varphi\left(u_{n_{i}}\right) \\
& -\left\langle u_{t}-u_{n_{i}}, \frac{u_{n_{i}}-x_{n_{i}}}{r_{n_{i}}}\right\rangle+\Theta\left(u_{t}, u_{n_{i}}\right) .
\end{aligned}
$$

Since $\left\|u_{n_{i}}-x_{n_{i}}\right\| \rightarrow 0$, we have $\left\|A u_{n_{i}}-A x_{n_{i}}\right\| \rightarrow 0$. Further, from the monotonicity of $A$, we have $\left\langle u_{t}-u_{n_{i}}, A u_{t}-A u_{n_{i}}\right\rangle \geq 0$. So, from (H4), the weakly lower semicontinuity of $\varphi, \frac{u_{n_{i}}-x_{n_{i}}}{r_{n_{i}}} \rightarrow 0$ and $u_{n_{i}} \rightarrow w$, we have

$$
\left\langle u_{t}-w, A u_{t}\right\rangle \geq-\varphi\left(u_{t}\right)+\varphi(w)+\Theta\left(u_{t}, w\right),
$$

as $i \rightarrow \infty$. From (H1), (H4) and (3.19), we also have

$$
\begin{aligned}
0 & =\Theta\left(u_{t}, u_{t}\right)+\varphi\left(u_{t}\right)-\varphi\left(u_{t}\right) \\
& \leq t \Theta\left(u_{t}, y\right)+(1-t) \Theta\left(u_{t}, w\right)+t \varphi(y)+(1-t) \varphi(w)-\varphi\left(u_{t}\right) \\
& =t\left[\Theta\left(u_{t}, y\right)+\varphi(y)-\varphi\left(u_{t}\right)\right]+(1-t)\left[\Theta\left(u_{t}, w\right)+\varphi(w)-\varphi\left(u_{t}\right)\right] \\
& \leq t\left[\Theta\left(u_{t}, y\right)+\varphi(y)-\varphi\left(u_{t}\right)\right]+(1-t)\left\langle u_{t}-w, A u_{t}\right\rangle \\
& =t\left[\Theta\left(u_{t}, y\right)+\varphi(y)-\varphi\left(u_{t}\right)\right]+(1-t) t\left\langle y-w, A u_{t}\right\rangle,
\end{aligned}
$$

and hence

$$
0 \leq \Theta\left(u_{t}, y\right)+\varphi(\gamma)-\varphi\left(u_{t}\right)+(1-t)\left\langle y-w, A u_{t}\right\rangle .
$$

Letting $t \rightarrow 0$, we have, for each $y \in C$,

$$
0 \leq \Theta(w, y)+\varphi(\gamma)-\varphi(w)+\langle y-w, A w\rangle .
$$


This implies that $w \in G M E P$.

We shall show $w \in \cap_{i=1}^{N} \operatorname{Fix}\left(S_{i}\right)$. To see this, we observe that we may assume (by passing to a further subsequence if necessary)

$$
\lambda_{n_{m}, k} \rightarrow \lambda_{k} \in(0,1)(k=1,2, \ldots, N) .
$$

Let $W$ be the $W$-mapping generated by $S_{1}, \ldots, S_{N}$ and $\lambda_{1}, \ldots, \lambda_{N}$. Then by Proposition 2.3, we have, for every $x \in H$,

$$
W_{n_{m}} x \rightarrow W x .
$$

Moreover, from Proposition 2.2 it follows that $\operatorname{Fix}(W)=\cap_{i=1}^{N} \operatorname{Fix}\left(S_{i}\right)$. Assume that $w \notin \cap_{i=1}^{N} \operatorname{Fix}\left(S_{i}\right)$; then $w \neq W w$. Since $w \in G M E P$, in terms of $\left\|x_{n}-W_{n} u_{n}\right\| \rightarrow 0$ and Opial's property of a Hilbert space, we conclude from (3.20) that

$$
\begin{aligned}
\liminf _{m \rightarrow \infty}\left\|x_{n_{m}}-w\right\| & <\liminf _{m \rightarrow \infty}\left\|x_{n_{m}}-W w\right\| \\
& \leq \liminf _{m \rightarrow \infty}\left(\left\|x_{n_{m}}-W_{n_{m}} u_{n_{m}}\right\|+\left\|W_{n_{m}} u_{n_{m}}-W_{n_{m}} w\right\|+\left\|W_{n_{m}} w-W w\right\|\right) \\
& =\liminf _{m \rightarrow \infty}\left\|W_{n_{m}} u_{n_{m}}-W_{n_{m}} w\right\| \\
& \leq \liminf _{m \rightarrow \infty}\left\|u_{n_{m}}-w\right\| \\
& =\liminf _{m \rightarrow \infty}\left\|T_{r_{m_{m}}}^{(\Theta)}\left(x_{n_{m}}-r_{n_{m}} A x_{n_{m}}\right)-T_{r_{n_{m}}}^{(\Theta, \varphi)}\left(w-r_{n_{m}} A w\right)\right\| \\
& \leq \liminf _{m \rightarrow \infty}\left\|\left(x_{n_{m}}-r_{n_{m}} A x_{n_{m}}\right)-\left(w-r_{n_{m}} A w\right)\right\| \\
& =\liminf _{m \rightarrow \infty}\left\|x_{n_{m}}-w-r_{n_{m}}\left(A x_{n_{m}}-A w\right)\right\| \\
& \leq \liminf _{m \rightarrow \infty}\left\|x_{n_{m}}-w\right\|,
\end{aligned}
$$

due to the $\delta$-inverse strong monotonicity of $A$. This is a contradiction. So, we get $\quad w \in \bigcap_{i=1}^{N} \operatorname{Fix}\left(S_{i}\right)$. Therefore $w \in \bigcap_{i=1}^{N} \operatorname{Fix}\left(S_{i}\right) \cap G M E P$. Since $x^{*}=P_{\bigcap_{i=1}^{N} \operatorname{Fix}\left(S_{i}\right) \cap G M E P}(I-\mu F+\gamma f) x^{*}$, we have

$$
\begin{aligned}
\limsup _{n \rightarrow \infty}\left\langle(\mu F-\gamma f) x^{*}, x^{*}-x_{n}\right\rangle & =\limsup _{n \rightarrow \infty}\left\langle(\mu F-\gamma f) x^{*}, x^{*}-u_{n}\right\rangle \\
& =\lim _{i \rightarrow \infty}\left\langle(\mu F-\gamma f) x^{*}, x^{*}-u_{n_{i}}\right\rangle \\
& =\left\langle(\mu F-\gamma f) x^{*}, x^{*}-w\right\rangle \leq 0 .
\end{aligned}
$$

Finally, we prove that $\left\{x_{n}\right\}$ and $\left\{u_{n}\right\}$ converge strongly to $x^{*}$. From (3.1), utilizing Lemmas 2.5 and 2.7 we have

$$
\begin{aligned}
&\left\|x_{n+1}-x^{*}\right\|^{2} \\
&=\left\|\alpha_{n}\left(\gamma f\left(x_{n}\right)-\mu F x^{*}\right)+\beta_{n}\left(x_{n}-x^{*}\right)+\left(\left(1-\beta_{n}\right) I-\alpha_{n} \mu F\right) W_{n} u_{n}-\left(\left(1-\beta_{n}\right) I-\alpha_{n} \mu F\right) W_{n} x^{*}\right\|^{2} \\
& \leq\left\|\beta_{n}\left(x_{n}-x^{*}\right)+\left(\left(1-\beta_{n}\right) I-\alpha_{n} \mu F\right) W_{n} u_{n}-\left(\left(1-\beta_{n}\right) I-\alpha_{n} \mu F\right) W_{n} x^{*}\right\|^{2} \\
&+2 \alpha_{n}\left\langle\gamma f\left(x_{n}\right)-\mu F x^{*}, x_{n+1}-x^{*}\right\rangle \\
& \leq {\left[\beta_{n}\left\|x_{n}-x^{*}\right\|+\left\|\left(\left(1-\beta_{n}\right) I-\alpha_{n} \mu F\right) W_{n} u_{n}-\left(\left(1-\beta_{n}\right) I-\alpha_{n} \mu F\right) W_{n} x^{*}\right\|\right]^{2} } \\
&+2 \alpha_{n}\left\langle\gamma f\left(x_{n}\right)-\mu F x^{*}, x_{n+1}-x^{*}\right\rangle \\
& \leq {\left[\beta_{n}\left\|x_{n}-x^{*}\right\|+\left\|\left(\left(1-\beta_{n}\right) I-\frac{\alpha_{n}}{1-\beta_{n}} \mu F\right) W_{n} u_{n}-\left(I-\frac{\alpha_{n}}{1-\beta_{n}} \mu F\right) W_{n} x^{*}\right\|\right]^{2} } \\
&+2 \alpha_{n} \gamma\left\langle f\left(x_{n}\right)-f\left(x^{*}\right), x_{n+1}-x^{*}\right\rangle+2 \alpha_{n}\left\langle\gamma f\left(x^{*}\right)-\mu F x^{*}, x_{n+1}-x^{*}\right\rangle \\
& \leq {\left[\beta_{n}\left\|x_{n}-x^{*}\right\|+\left(1-\beta_{n}\right)\left(1-\frac{\alpha_{n}}{1-\beta_{n}} \tau\right)\left\|x_{n}-x^{*}\right\|\right]^{2} } \\
&+2 \alpha_{n} \gamma \rho\left\|x_{n}-x^{*}\right\|\left\|x_{n+1}-x^{*}\right\|+2 \alpha_{n}\left\langle\gamma f\left(x^{*}\right)-\mu F x^{*}, x_{n+1}-x^{*}\right\rangle \\
& \leq\left(1-\alpha_{n} \tau\right)^{2}\left\|x_{n}-x^{*}\right\|^{2}+\alpha_{n} \gamma \rho\left(\left\|x_{n}-x^{*}\right\|^{2}+\left\|x_{n+1}-x^{*}\right\|^{2}\right) \\
&+2 \alpha_{n}\left\langle\gamma f\left(x^{*}\right)-\mu F x^{*}, x_{n+1}-x^{*}\right\rangle \\
&=\left(1-\alpha_{n} \tau\right)^{2}\left\|x_{n}-x^{*}\right\|^{2}+\alpha_{n} \gamma \rho\left\|x_{n}-x^{*}\right\|^{2}+\alpha_{n} \gamma \rho\left\|x_{n+1}-x^{*}\right\|^{2} \\
&+2 \alpha_{n}\left\langle\gamma f\left(x^{*}\right)-\mu F x^{*}, x_{n+1}-x^{*}\right\rangle .
\end{aligned}
$$


This implies that

$$
\begin{aligned}
\left\|x_{n+1}-x^{*}\right\|^{2} \leq & \frac{\left(1-\alpha_{n} \tau\right)^{2}+\alpha_{n} \gamma \rho}{1-\alpha_{n} \gamma \rho}\left\|x_{n}-x^{*}\right\|^{2}+\frac{2 \alpha_{n}}{1-\alpha_{n} \gamma \rho}\left\langle\gamma f\left(x^{*}\right)-\mu F x^{*}, x_{n+1}-x^{*}\right\rangle \\
= & {\left[1-\frac{2(\tau-\gamma \rho) \alpha_{n}}{1-\alpha_{n} \gamma \rho}\right]\left\|x_{n}-x^{*}\right\|^{2}+\frac{\left(\alpha_{n} \tau\right)^{2}}{1-\alpha_{n} \gamma \rho}\left\|x_{n}-x^{*}\right\|^{2} } \\
& +\frac{2 \alpha_{n}}{1-\alpha_{n} \gamma \rho}\left\langle\gamma f\left(x^{*}\right)-\mu F x^{*}, x_{n+1}-x^{*}\right\rangle \\
\leq & {\left[1-\frac{2(\tau-\gamma \rho) \alpha_{n}}{1-\alpha_{n} \gamma \rho}\right]\left\|x_{n}-x^{*}\right\|^{2}+\frac{2(\tau-\gamma \rho) \alpha_{n}}{1-\alpha_{n} \gamma \rho} } \\
& \left.\times\left\{\frac{\left(\alpha_{n} \tau^{2}\right) M_{1}}{2(\tau-\gamma \rho)}+\frac{1}{\tau-\gamma \rho} \gamma f\left(x^{*}\right)-\mu F x^{*}, x_{n+1}-x^{*}\right\rangle\right\} \\
= & \left(1-\delta_{n}\right)\left\|x_{n}-x^{*}\right\|^{2}+\delta_{n} \sigma_{n},
\end{aligned}
$$

where $M_{1}=\sup \left\{\left\|x_{n}-p\right\|^{2}: \quad n \geq 1\right\}, \quad \delta_{n}=\frac{2(\tau-\gamma \rho) \alpha_{n}}{1-\alpha_{n} \gamma \rho} \quad$ and $\left.\sigma_{n}=\frac{\left(\alpha_{n} \tau^{2}\right) M_{1}}{2(\tau-\gamma p)}+\frac{1}{\tau-\gamma \rho} \gamma f\left(x^{*}\right)-\mu F x^{*}, x_{n+1}-x^{*}\right\rangle$. It is easy to see that $\delta_{n} \rightarrow 0, \sum_{n=1}^{\infty} \delta_{n}=\infty$ and $\lim \sup _{n \rightarrow \infty} \sigma_{n} \leq 0$. Hence, by Lemma 2.4, the sequence $\left\{x_{n}\right\}$ converges strongly to $x^{*}$. Consequently, we can obtain from $\left\|x_{n}-u_{n}\right\| \rightarrow 0$ that $\left\{u_{n}\right\}$ also converges strongly to $x^{*}$. This completes the proof. $\square$

\section{Remark 3.1.}

(i) The new technique of argument is applied to derive our Theorem 3.1. For instance, Lemma 2.7 for deriving the convergence of hybrid steepest-descent method plays an important role in proving the strong convergence of the sequences $\left\{x_{n}\right\},\left\{u_{n}\right\}$ in our Theorem 3.1. In addition, utilizing Proposition 2.1 and $r_{n+1}-r_{n} \rightarrow 0$ we can obtain $\lim _{n \rightarrow \infty}\left\|T_{r_{n+1}}^{(\Theta, \varphi)}\left(x_{n}-r_{n} A x_{n}\right)-T_{r_{n}}^{(\Theta, \varphi)}\left(x_{n}-r_{n} A x_{n}\right)\right\|=0$.

(ii) In order to show $w \in \cap_{i=1}^{N} \operatorname{Fix}\left(S_{i}\right)$, the proof of Theorem 3.2 [10] directly asserts that $\left\|u_{n}-W_{n} u_{n}\right\| \rightarrow 0(n \rightarrow \infty)$ implies $\left\|u_{n_{j}}-W_{n} u_{n_{j}}\right\| \rightarrow 0(j \rightarrow \infty)$ for all $n$. Actually, this assertion seems impossible under their assumptions imposed on $\left\{\lambda_{n, i}\right\}_{i=1}^{N}$. However, following Colao, Marino and Xu's Step 7 of the proof in [[14], Theorem 3.1] and utilizing Proposition 2.3 (i.e., Lemma 2.8 in [14]), we successively derive $w \in \cap_{i=1}^{N} \operatorname{Fix}\left(S_{i}\right)$ by the condition $\left\{\lambda_{n, i}\right\}_{i=1}^{N} \subset[a, b]$ with $0<a \leq b<1$.

Theorem 3.2. Let $C$ be a nonempty closed convex subset of a real Hilbert space $H$. Let $A: H \rightarrow H$ be $\delta$-inverse strongly monotone, $\Theta: C \times C \rightarrow \mathbf{R}$ be a bifunction satisfying assumptions ( $\mathrm{H} 1)-(\mathrm{H} 4)$ and $\phi: C \rightarrow \mathbf{R}$ be a lower semicontinuous and convex function with restriction (A1) or (A2) such that GMEP $\neq \varnothing$. Let $F: H \rightarrow H$ be a $\kappa$-Lipschitzian and $\eta$-strongly monotone operator with constants $\kappa, \eta>0$ and $f: H \rightarrow$ $H$ a $\rho$-Lipschitzian mapping with constant $\rho \geq 0$. Let $0<\mu<2 \eta / \kappa^{2}$ and $0 \leq \gamma \rho<\tau$, where $\tau=1-\sqrt{1-\mu\left(2 \eta-\mu \kappa^{2}\right)}$. Suppose $\left\{\alpha_{n}\right\}$ and $\left\{\beta_{n}\right\}$ are two sequences in $(0,1)$ and $\left\{r_{n}\right\}$ is a sequence in $(0,2 \delta]$. Given $x_{1} \in H$ arbitrarily, suppose the sequences $\left\{x_{n}\right\}$ and $\left\{u_{n}\right\}$ are generated iteratively by

$$
\left\{\begin{array}{l}
\Theta\left(u_{n}, \gamma\right)+\varphi(y)-\varphi\left(u_{n}\right)+\left\langle A x_{n}, y-u_{n}\right\rangle+\frac{1}{r_{n}}\left\langle y-u_{n}, u_{n}-x_{n}\right\rangle \geq 0, \quad \forall y \in C, \\
x_{n+1}=\alpha_{n} \gamma f\left(x_{n}\right)+\beta_{n} x_{n}+\left(\left(1-\beta_{n}\right) I-\alpha_{n} \mu F\right) u_{n}, \quad \forall n \geq 1,
\end{array}\right.
$$

where the sequences $\left\{\alpha_{n}\right\},\left\{\beta_{n}\right\},\left\{r_{n}\right\}$ satisfy the conditions: 
(i) $\lim _{n \rightarrow \infty} \alpha_{n}=0$ and $\sum_{n=1}^{\infty} \alpha_{n}=\infty$;

(ii) $0<\lim \inf _{n \rightarrow \infty} \beta_{n} \leq \lim \sup _{n \rightarrow \infty} \beta_{n}<1$;

(iii) $0<\lim \inf _{n \rightarrow \infty} r_{n} \leq \lim \sup _{n \rightarrow \infty} r_{n}<2 \delta$ and $\lim _{n \rightarrow \infty}\left(r_{n+1}-r_{n}\right)=0$.

Then both $\left\{x_{n}\right\}$ and $\left\{u_{n}\right\}$ converge strongly to $x^{*} \in G M E P$, where $x^{*}=P_{G M E P}(I-\mu F+$ $\gamma f f x^{*}$.

Proof. Put $S_{i} x=x$ for all $i=1,2, \ldots, N$ and $x \in H$ and take the finite family of sequences $\left\{\lambda_{n, i}\right\}_{i=1}^{N}$ in $[a, b]$ with $0<a \leq b<1$ such that $\lim _{n \rightarrow \infty}\left(\lambda_{n+1, i}-\lambda_{n, i}\right)=0$ for all $i=1,2, \ldots, N$. In this case, the $W$-mapping $W_{n}$ generated by $S_{1}, \ldots, S_{N}$ and $\lambda_{n, 1}$, $\lambda_{n, 2}, \ldots, \lambda_{n, N}$, is the identity mapping $I$ of $H$. It is easy to see that all conditions of Theorem 3.1 are satisfied. Thus, the desired result follows from Theorem 3.1. $\square$

Theorem 3.3. Let $H$ be a real Hilbert space. Let $\left\{S_{i}\right\}_{i=1}^{N}$ be a finite family of nonexpansive mappings on $H$ such that $\cap_{i=1}^{N} \operatorname{Fix}\left(S_{i}\right) \neq \emptyset$. Let $F: H \rightarrow H$ be a $\kappa$-Lipschitzian and $\eta$-strongly monotone operator with constants $\kappa, \eta>0$ and $f: H \rightarrow H$ a $\rho$-Lipschitzian mapping with constant $\rho \geq 0$. Let $0<\mu<2 \eta / \kappa^{2}$ and $0 \leq \gamma \rho<\tau$, where $\tau=1-\sqrt{1-\mu\left(2 \eta-\mu \kappa^{2}\right)}$. Suppose $\left\{\alpha_{n}\right\}$ and $\left\{\beta_{n}\right\}$ are two sequences in $(0,1)$ and $\left\{\lambda_{n, i}\right\}_{i=1}^{N}$ is a sequence in $[a, b]$ with $0<a \leq b<1$. For every $n \geq 1$, let $W_{n}$ be the $W$ mapping generated by $S_{1}, \ldots, S_{N}$ and $\lambda_{n, 1}, \lambda_{n, 2}, \ldots, \lambda_{n, N}$. Given $x_{1} \in H$ arbitrarily, let $\left\{x_{n}\right\}$ be a sequence generated by

$$
x_{n+1}=\alpha_{n} \gamma f\left(x_{n}\right)+\beta_{n} x_{n}+\left(\left(1-\beta_{n}\right) I-\alpha_{n} \mu F\right) W_{n} x_{n}, \quad \forall n \geq 1,
$$

where the sequences $\left\{\alpha_{n}\right\},\left\{\beta_{n}\right\}$ and the finite family of sequences $\left\{\lambda_{n, i}\right\}_{i=1}^{N}$ satisfy the conditions:

(i) $\lim _{n \rightarrow \infty} \alpha_{n}=0$ and $\sum_{n=1}^{\infty} \alpha_{n}=\infty$;

(ii) $0<\lim \inf _{n \rightarrow \infty} \beta_{n} \leq \lim \sup _{n \rightarrow \infty} \beta_{n}<1$;

(iii) $\lim _{n \rightarrow \infty}\left(\lambda_{n+1, i}-\lambda_{n, i}\right)=0$ for all $i=1,2, \ldots, N$.

Then $\left\{x_{n}\right\}$ converges strongly to $x^{*} \in \cap_{i=1}^{N} \operatorname{Fix}\left(S_{i}\right)$, where $x^{*}=P_{\bigcap_{i=1}^{N} \operatorname{Fix}\left(S_{i}\right)}(I-\mu F+\gamma f) x^{*}$.

Proof. Put $C=H$ and $r_{n}=1$, and take $\Theta(x, y)=0, A x=0$ and $\phi(x)=0$ for all $x, y \in$ $H$. Then $\Theta: H \times H \rightarrow \mathbf{R}$ is a bifunction satisfying assumptions (H1)-(H4) and $\phi: H \rightarrow$ $\mathbf{R}$ is a lower semicontinuous and convex function with restriction (A1). Moreover the mapping $A: H \rightarrow H$ is $\delta$-inverse strongly monotone for any $\delta>\frac{1}{2}$. In this case, from Theorem 3.1 we deduce that $u_{n}=x_{n}, 0<\lim _{\inf _{n \rightarrow \infty}} r_{n} \leq \lim \sup _{n \rightarrow \infty} r_{n}<2 \delta$ and $\lim _{n \rightarrow \infty}\left(r_{n+1}-r_{n}\right)=0$. Beyond question, all conditions of Theorem 3.1 are satisfied. Therefore the conclusion follows.

\section{Acknowledgements}

Lu-Chuan Ceng was partially supported by National Science Foundation of China (11071169), Innovation Program of Shanghai Municipal Education Commission (09ZZ133) and Leading Academic Discipline Project of Shanghai Normal University (DZL707), Sy-Ming Guu was partially supported by the grant NSC 97-2221-E-155-041-MY3, and Jen-Chih Yao was partially supported by the grant NSC 99-2221-E-037-007-MY3. 
Yuan-Ze University, Chung-Li, Taoyuan Hsien 330, Taiwan ${ }^{4}$ Center for General Education, Kaohsiung Medical University, Kaohsiung 807, Taiwan

\section{Authors' contributions}

LC conceived of the study and drafted the manuscript initially. SM participated in its design, coordination and finalized the manuscript. JC outlined the scope and design of the study. All authors read and approved the final manuscript.

\section{Competing interests}

The authors declare that they have no competing interests.

\section{Received: 13 September 2011 Accepted: 6 June 2012 Published: 6 June 2012}

\section{References}

1. Blum, E, Oettli, W: From optimization and variational inequalities to equilibrium problems. Math Stud. 63, 123-145 (1994)

2. Aslam Noor, M, Oettli, W: On general nonlinear complementarity problems and quasi equilibria. Mathematiche (Catania). 49, 313-331 (1994)

3. Combettes, PL, Hirstoaga, SA: Equilibrium problems in Hilbert spaces. J Nonlinear Convex Anal. 6, 117-136 (2005)

4. Peng, JW, Yao, JC: A new hybrid-extragradient method for generalized mixed equilibrium problems, fixed point problems and variational inequality problems. Taiwan J Math. 12, 1401-1432 (2008)

5. Ceng, LC, Yao, JC: A hybrid iterative scheme for mixed equilibrium problems and fixed point problems. J Comput App Math. 214, 186-201 (2008). doi:10.1016/j.cam.2007.02.022

6. Ceng, LC, Yao, JC: A relaxed extragradient-like method for a generalized mixed equilibrium problem, a general system of generalized equilibria and a fixed point problem. Nonlinear Anal. 72, 1922-1937 (2010). doi:10.1016/j.na.2009.09.033

7. Combettes, PL, Hirstoaga, SA: Equilibrium programming in Hilbert spaces. J Nonlinear Convex Anal. 6, 117-136 (2005)

8. Chadli, O, Konnov, IV, Yao, JC: Descent methods for equilibrium problems in a Banach space. Comput Math Appl. 48, 609-616 (2004). doi:10.1016/j.camwa.2003.05.011

9. Zeng, LC, Wu, SY, Yao, JC: Generalized KKM theorem with applications to generalized minimax inequalities and generalized equilibrium problems. Taiwan J Math. 10, 1497-1514 (2006)

10. Yao, Y, Aslam Noor, M, Liou, YC: On iterative methods for equilibrium problems. Nonlinear Anal. 70, 497-509 (2009). doi:10.1016/j.na.2007.12.021

11. Plubtieng, S, Punpaeng, R: A general iterative method for equilibrium problems and fixed point problems in Hilbert spaces. J Math Anal Appl. 336, 455-469 (2007). doi:10.1016/j.jmaa.2007.02.044

12. Takahashi, S, Takahashi, W: Viscosity approximation methods for equilibrium problems and fixed point problems in Hilbert spaces. J Math Anal Appl. 331, 506-515 (2007). doi:10.1016/j.jmaa.2006.08.036

13. Takahashi, S, Takahashi, W: Strong convergence theorem for a generalized equilibrium problem and a nonexpansive mapping in a Hilbert space. Nonlinear Anal. 69, 1025-1033 (2008). doi:10.1016/j.na.2008.02.042

14. Colao, V, Marino, G, Xu, HK: An iterative method for finding common solutions of equilibrium and fixed point problems. J Math Anal Appl. 344, 340-352 (2008). doi:10.1016/j.jmaa.2008.02.041

15. Ceng, LC, Yao, JC: Hybrid viscosity approximation schemes for equilibrium problems and fixed point problems of infinitely many nonexpansive mappings. Appl Math Comput. 198, 729-741 (2008). doi:10.1016/j.amc.2007.09.011

16. Ceng, LC, Al-Homidan, S, Ansari, QH, Yao, JC: An iterative scheme for equilibrium problems and fixed point problems of strict pseudo-contraction mappings. J Comput Appl Math. 223, 967-974 (2009). doi:10.1016/j.cam.2008.03.032

17. Ceng, LC, Schaible, S, Yao, JC: Implicit iteration scheme with perturbed mapping for equilibrium problems and fixed point problems of finitely many nonexpansive mappings. J Optim Theory Appl. 139, 403-418 (2008). doi:10.1007/ s10957-008-9361-y

18. Ceng, LC, Wong, NC: Viscosity approximation methods for equilibrium problems and fixed point problems of nonlinear semigroups. Taiwan J Math. 13(5), 1497-1513 (2009)

19. $\mathrm{Xu}, \mathrm{HK}, \mathrm{Kim}, \mathrm{TH}$ : Convergence of hybrid steepest-descent methods for variational inequalities. J Optim Theory Appl. 119, 185-201 (2003)

20. Yamada, I: The hybrid steepest descent method for the variational inequality problems over the intersection of fixedpoint sets of nonexpansive mappings. In: Butnariu D, Censor Y, Reich V (eds.) Inherently Parallel Algorithms in Feasibility and Optimization and Their Applications. pp. 473-504. North-Holland: Amsterdam (2001)

21. Suzuki, T: Strong convergence of Krasnoselskii and Mann's type sequences for one-parameter nonexpansive semigroups without Bochner integrals. J Math Anal Appl. 305, 227-239 (2005). doi:10.1016/j.jmaa.2004.11.017

22. Atsushiba, S, Takahashi, W: Strong convergence theorems for a finite family of nonexpansive mappings and applications. Indian J Math. 41, 435-453 (1999)

23. Takahashi, W, Shimoji, K: Convergence theorems for nonexpansive mappings and feasibility problems. Math Comput Modelling. 32, 1463-1471 (2000). doi:10.1016/S0895-7177(00)00218-1

24. Zeng, LC, Wong, NC, Yao, JC: Convergence analysis of modified hybrid steepest-descent methods with variable parameters for variational inequalities. J Optim Theory Appl. 132, 51-69 (2007). doi:10.1007/s10957-006-9068-x

25. Ceng, LC, Cubiotti, P, Yao, JC: Strong convergence theorems for finitely many nonexpansive mappings and applications. Nonlinear Anal. 67, 1464-1473 (2007). doi:10.1016/j.na.2006.06.055

26. Zeng, LC, Yao, JC: Implicit iteration scheme with perturbed mapping for common fixed points of a finite family of nonexpansive mappings. Nonlinear Anal. 64, 2507-2515 (2006). doi:10.1016/j.na.2005.08.028

27. Yao, Y: A general iterative method for a finite family of nonexpansive mappings. Nonlinear Anal. 66, 2676-2687 (2007). doi:10.1016/j.na.2006.03.047

28. Moudafi, A: Viscosity approximation methods for fixed-points problems. J Math Anal Appl. 241(1), 46-55 (2000). doi:10.1006/jmaa.1999.6615 
29. Xu, HK: Viscosity approximation methods for nonexpansive mappings. J Math Anal Appl. 298, 279-291 (2004). doi:10.1016/j.jmaa.2004.04.059

30. Cianciaruso, F, Colao, V, Muglia, L, Xu, HK: On an implicit hierarchical fixed point approach to variational inequalities. Bull Aust Math Soc. 80(1), 117-124 (2009). doi:10.1017/50004972709000082

31. Ceng, LC, Xu, HK, Yao, JC: The viscosity approximation method for asymptotically nonexpansive mappings in Banach spaces. Nonlinear Anal. 69, 1402-1412 (2008). doi:10.1016/j.na.2007.06.040

32. Ceng, LC, Huang, S, Petrusel, A: Weak convergence theorem by a modified extragradient method for nonexpansive mappings and monotone mappings. Taiwan J Math. 13(1), 225-238 (2009)

33. Ceng, LC, Petrusel, A, Lee, C, Wong, MM: Two extragradient approximation methods for variational inequalities and fixed point problems of strict pseudo-contractions. Taiwan J Math. 13(2A), 607-632 (2009)

34. Ceng, LC, Huang, S, Liou, YC: Hybrid proximal point algorithms for solving constrained minimization problems in Banach spaces. Taiwan J Math. 13(2B), 805-820 (2009)

35. Ceng, LC, Guu, SM, Yao, JC: A general iterative method with strongly positive operators for general variational inequalities. Comput Math Appl. 59, 1441-1452 (2010). doi:10.1016/j.camwa.2009.11.007

36. Takahashi, W: Nonlinear Functional Analysis: Fixed Point Theory and Its Applications. Yokohama Publishers, Yokohama (2000)

doi:10.1186/1687-1812-2012-92

Cite this article as: Ceng et al:: Hybrid iterative method for finding common solutions of generalized mixed equilibrium and fixed point problems. Fixed Point Theory and Applications 2012 2012:92.

\section{Submit your manuscript to a SpringerOpen ${ }^{\odot}$} journal and benefit from:

- Convenient online submission

Rigorous peer review

- Immediate publication on acceptance

- Open access: articles freely available online

- High visibility within the field

- Retaining the copyright to your article

Submit your next manuscript at $\boldsymbol{\triangleright}$ springeropen.com 